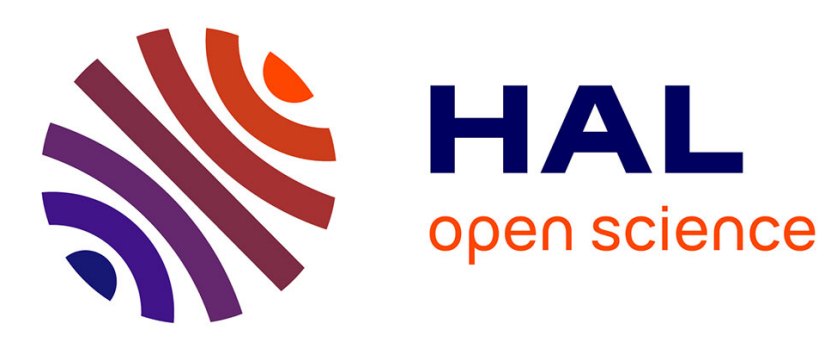

\title{
Modelling force-length-activation relationships of wrist and finger extensor muscles
}

Benjamin Goislard de Monsabert, Hugo Hauraix, Mathieu Caumes, Alexis Herbaut, Eric Berton, Laurent Vigouroux

\section{- To cite this version:}

Benjamin Goislard de Monsabert, Hugo Hauraix, Mathieu Caumes, Alexis Herbaut, Eric Berton, et al.. Modelling force-length-activation relationships of wrist and finger extensor muscles. Medical and Biological Engineering and Computing, 2020, 58 (10), pp.2531-2549. 10.1007/s11517-020-02239-0 . hal-02942601

\section{HAL Id: hal-02942601 \\ https://hal.science/hal-02942601}

Submitted on 18 Sep 2020

HAL is a multi-disciplinary open access archive for the deposit and dissemination of scientific research documents, whether they are published or not. The documents may come from teaching and research institutions in France or abroad, or from public or private research centers.
L'archive ouverte pluridisciplinaire HAL, est destinée au dépôt et à la diffusion de documents scientifiques de niveau recherche, publiés ou non, émanant des établissements d'enseignement et de recherche français ou étrangers, des laboratoires publics ou privés. 


\section{Modelling Force-Length-Activation relationships of wrist and finger extensor muscles}

\section{Title Page}

\section{Authors and affiliations}

Benjamin GOISLARD DE MONSABERT ${ }^{1 *}$, Hugo HAURAIX ${ }^{1}$, Mathieu CAUMES $^{1}$, Alexis HERBAUT $^{2}$, Eric BERTON ${ }^{1}$, Laurent VIGOUROUX ${ }^{1}$

${ }^{1}$ Aix-Marseille University, CNRS, ISM, Marseille, France.

${ }^{2}$ Department of Movement Sciences, Decathlon SportsLab, Villeneuve d'Ascq, France.

*Corresponding author:

Benjamin GOISLARD DE MONSABERT

UMR 7287 CNRS \& Aix-Marseille University

Faculté des Sciences du Sport, CP 910

163, av. de Luminy,

F-13288 Marseille cedex 09 (FRANCE)

Phone: (+33) 491759655

Fax: (+33) 491172252

email: benjamin.goislard-de-monsabert@univ-amu.fr

Number of words: 11348 (from Title Page to Figure Legends, included)

Number of words of the abstract: 196

Number of figures: 8

Number of tables: 1 


\begin{abstract}
The wrist and finger extensors play a crucial role in the muscle coordination during grasping tasks. Nevertheless, few data are available regarding their force-generating capacities. The objective of this study was to provide a model of the Force-Length-Activation relationships of the hand extensors using non-invasive methods. The extensor carpi radialis (ECR) and the extensor digitorum communis (EDC) were studied as representative of wrist and finger extensors. Ten participants performed isometric extension force-varying contractions in different postures on an ergometer recording resultant moment. The joint angle, the myotendinous junction displacement and activation were synchronously tracked using motion capture, ultrasound, and electromyography. Muscle force was estimated via a musculoskeletal model using the measured joint angle and moment. The Force-Length-Activation relationship was then obtained by fitting a force-length model at different activation levels to the measured data. The obtained relationships agreed with previously reported data regarding muscle architecture, sarcomere length and activation-dependent shift of optimal length. Muscle forces estimated from kinematics and electromyography using the Force-Length-Activation relationships were comparable, below $15 \%$ differences, to those estimated from moment via the musculoskeletal model. The obtained quantitative data provides a new insight into the different muscle mechanics of finger and wrist extensors.
\end{abstract}




\section{Graphical abstract}
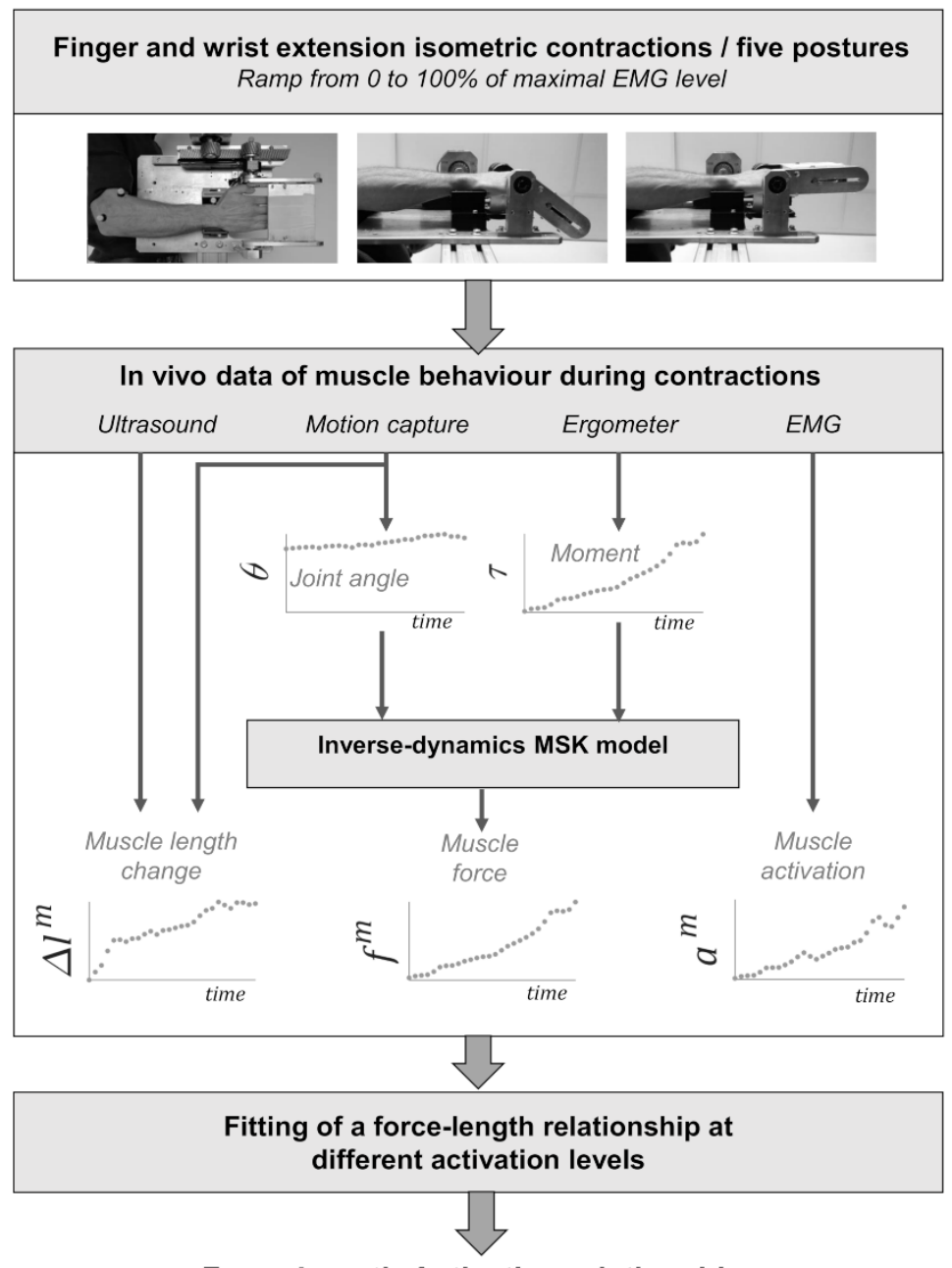

Force-Length-Activation relationships

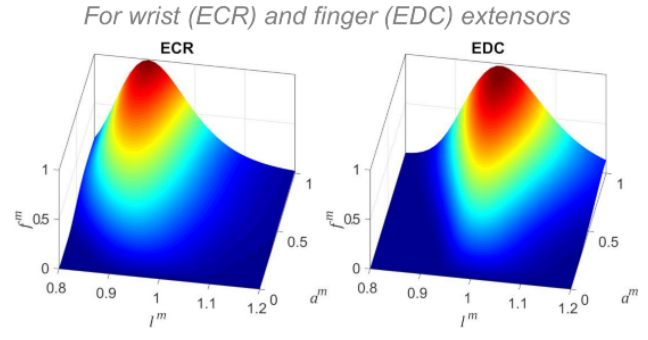

By combining in vivo data (kinematics, dynamometry, electromyography, ultrasonography) during isometric force-varying contractions with musculoskeletal modelling, the Force-LengthActivation relationships of both finger and wrist extensors were obtained. The results provided a new insight into the role of hand extensors in the generation and control of hand movements.

\section{Keywords}

Hand, Muscle Contraction, Ultrasonography, Electromyography, Biological Models 


\section{Glossary of terms}

\begin{tabular}{|c|c|}
\hline AVE & "Average" EMG-driven model \\
\hline ECR & Extensor carpi radialis \\
\hline ECRB & Extensor carpi radialis brevis \\
\hline ECRL & Extensor carpi radialis longus \\
\hline EDC & Extensor digitorum communis \\
\hline EDCI & Index finger compartment of extensor digitorum communis \\
\hline EMG & Electromyography \\
\hline IND & "Individual” EMG-driven model \\
\hline $\mathrm{MCP}$ & Metacarpophalangeal \\
\hline MSK & Musculoskeletal \\
\hline NoFL & "No Force-Length" EMG-driven model \\
\hline PCSA & Physiological cross-sectional area \\
\hline RMSE & Root mean square error \\
\hline$a^{m}$ & Muscle activation level \\
\hline$\alpha_{i}$ & Constants describing the force-activation relationships \\
\hline$\beta$ & Skewness parameter of the force-length relationship of Otten \\
\hline$\gamma_{i}$ & Constants describing the length-force relationships in a ramp trial \\
\hline$\varepsilon^{m}$ & Muscle belly strain \\
\hline$F^{m}$ & Muscle force (Newtons) \\
\hline$f^{m}$ & Muscle force normalized by maximal value observed \\
\hline$F_{0}$ & Maximal isometric force at a given activation level \\
\hline$F_{0}^{\max }$ & Maximal isometric force at $100 \%$ of muscle activation \\
\hline$\theta_{w}$ & Wrist joint angle \\
\hline$\theta_{m c p}$ & Metacarpophalangeal joint angle \\
\hline
\end{tabular}




\begin{tabular}{|c|c|}
\hline$i_{a}$ & Index of architecture at a given activation level \\
\hline$i_{a}^{o p t}$ & Index of architecture at $100 \%$ of activation \\
\hline$L^{m}$ & Muscle belly length \\
\hline$l^{m}$ & Normalized muscle belly length \\
\hline$L_{1}^{m}$ & Muscle belly length at rest at a given joint angle (before contraction) \\
\hline$L_{r}^{m}$ & Reference muscle belly length, i.e. at rest in neutral posture \\
\hline$\Delta L^{m}$ & Muscle belly length change (current length vs at rest) \\
\hline$\Delta l^{m}$ & Muscle belly length change normalized by maximal value observed \\
\hline$L^{m t u}$ & Muscle-tendon unit length at a given joint angle \\
\hline$L_{r}^{m t u}$ & Reference muscle-tendon unit length measured in neutral posture \\
\hline$L_{0}$ & Optimal muscle length at a given activation level \\
\hline$L_{o p t}$ & Muscle optimal length at $100 \%$ of activation \\
\hline$l_{\text {opt }}$ & $\begin{array}{l}\text { Muscle optimal length at } 100 \% \text { of activation normalized by muscle length at rest } \\
\text { in neutral posture }\left(L_{r}^{m}\right)\end{array}$ \\
\hline$p$ & Index for a given posture \\
\hline$\rho$ & Roundness parameter of the force-length relationship of Otten \\
\hline$t$ & Index for a time sample \\
\hline$\tau$ & Net joint moment measured by the ergometer \\
\hline$\omega$ & Width parameter of the force-length relationship of Otten \\
\hline
\end{tabular}




\section{Author biographies}
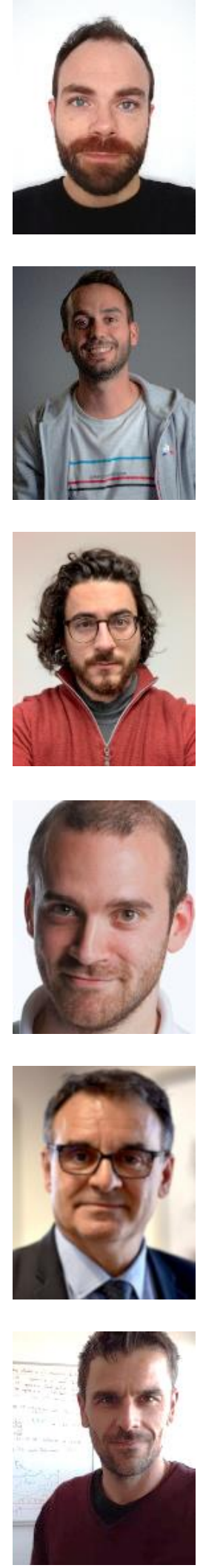

Benjamin Goislard de Monsabert, $\mathrm{PhD}$, completed his $\mathrm{PhD}$ (2014) at the Institute of Movement Sciences at Aix-Marseille University. He is Assistant Professor in musculoskeletal biomechanics since 2018 .

Hugo Hauraix, PhD, holds a PhD (2015) from the Movement, Interactions, Performance laboratory at the University of Nantes and is now working as a Data Analyst at Decathlon, sports retailer.

Mathieu Caumes, MSc, is a $\mathrm{PhD}$ candidate at the Institute of Movement Sciences at the Aix-Marseille University. His research focuses on wrist and hand biomechanics during grasping tasks.

Alexis Herbaut, $\mathrm{PhD}$, received his $\mathrm{PhD}$ (2016) from University of Valenciennes et du Hainaut Cambrésis and is now working as Research and Development Engineer at Decathlon SportsLab.

Eric Berton, $\mathrm{PhD}$, is a full professor at the Aix-Marseille University since 2005. His research focuses on human movement with applications to various domains such as sports engineering and ageing.

Laurent Vigouroux, $\mathrm{PhD}$, is an Associate Professor at the AixMarseille University since 2006. His research focuses on biomechanics and motor control of the hand and upper limbs. 


\section{Introduction}

Hand movements and object manipulation are essential for daily living. Thanks to its complex skeletal system composed of 21 or more joints, the hand offers multiple degrees of freedom and large ranges of motion which are essential for adapting to the task constraints, e.g. object dimensions and shape. Conjointly with these skeletal properties, the hand is actuated by a complex network of muscles and tendons crossing different joints which inherently create mechanical couplings between the wrist and the fingers. The most important coupling results from the anatomy of hand extrinsic muscles, such as the flexor digitorum superficialis, which originate in the forearm and insert on the phalanges and therefore both act at the finger joints and the wrist. During grasping tasks, the action of extrinsic flexors produces the grip force but concomitantly creates wrist flexion moments which can only be balanced by hand extensors, including both wrist prime movers such as extensor carpi radialis longus (ECRL) and brevis (ECRB) and finger extrinsic muscles such as extensor digitorum communis (EDC) $[1,2]$. Although they are antagonist to finger flexion, the co-contraction of hand extensors during grasping tasks is mechanically necessary to balance the wrist; an original synergy which does not exist in nonmanipulative force production tasks [3]. Nevertheless, although the behaviour of wrist extensors have been studied through intra-operative experiments [4-6], little is known about finger extensors, such as EDC. Because of this lack of information, the biomechanical couplings between the wrist and the fingers remain poorly known and a phenomenon such as the loss of grip force for extreme wrist postures $[7]$ remains unclear. Providing data regarding the mechanical behaviour of both finger and wrist extensors is relevant to understand the generation and control of hand movements.

Since direct measurements of muscle force are ethically and technically difficult and only provide measurement for isolated relatively large tendons [8], musculoskeletal models have continuously been developed to provide estimates of the internal mechanics of the finger and the wrist [9-18]. Those models require anatomical data describing the trajectories and morphology of muscle-tendon units as well as the bone geometries to quantify how each muscle can contribute to the generation of a movement or a force [19-22]. Furthermore, the potential contribution of 
each muscle also varies with the joint configuration as the maximal force it can produce depends on its current length, as described by the well-known force-length relationship [23]. In the most advanced hand musculoskeletal models, the force-length relationship of each muscle is obtained by scaling a generic curve using cadaver data $[11,15,16,24]$. Although this is a well-accepted way of modelling, such models often require a scaling of certain muscle-tendon unit parameters so they are more representative of participant abilities, especially the maximal joint torques $[25$. 26]. Nevertheless, because of the complexity of the hand and wrist anatomy, only few data exist regarding the capacities of this musculoskeletal system [27] and the link between joint postures, muscle lengths and maximum forces remain poorly understood. Considering the important changes in muscle length that can be generated along the range of motion covered by hand joints [28], it appears crucial for biomechanical models to quantify how the force-generating capacities of finger and wrist muscles varies with joint position.

The in vivo force-length behaviour of hand muscles have mainly been described through measurements of sarcomere length based on laser diffraction $[4,5]$ or micro-endoscopy $[6]$ which are invasive techniques. Although they provided crucial information about the muscle mechanics, those studies were focused on specific muscles, ECRB and ECRL, and the level of muscle force was interpreted from the generic curve of the force-length relationship provided by Gordon et al. [23]. Based on protocols previously developed to study the elbow [29, 30] and lower limb joints [31, 32], Hauraix et al. [33] recently developed a new method to evaluate the Force-LengthActivation relationships of hand flexors using non-invasive techniques to assess muscle behaviour and strength among healthy participants. The methodology combined musculoskeletal modelling with in vivo measurements of net joint moments, joint posture, myotendinous displacement and electromyography (EMG). Their approach especially allows to consider how activation can modify the force-length relationship, such as the shift of optimal length toward longer length when activation decreases [34-36]. Allowing to understand the muscle mechanics at low activation is crucial to understand hand function since manipulation tasks can require a wide range of force levels, e.g. sewing or hammering, and the muscle coordination varies according to the task constraints [3]. Considering the balance of muscle capacities between flexors and extensors 
can influence the muscle coordination and might represent a risk factor in lateral epicondylalgia [37], providing a framework to analyse the force-generating capacities of those muscle groups appears necessary.

The objective of this study was thus to adapt the method developed by Hauraix et al. to model the Force-Length-Activation relationships of the extensors of the fingers and the wrist using noninvasive techniques. The obtained relationships were compared to previous findings regarding cadaveric muscle architecture, in vivo sarcomere length and activation-dependent shift of optimal length. EMG-driven models were then developed based on the Force-Length-Activation to estimate muscle forces from kinematics and electromyography. The estimates of these models were compared to those of an inverse-dynamics musculoskeletal model using dynamometric data as input.

\section{Methods}

The methodology developed for hand flexors [33] was adapted to investigate two muscle groups representative of wrist and finger extensors. The ECR group was assumed to represent the global behaviour of both ECRB and ECRL muscles that are extensors and radial deviators of the wrist. The EDC group was assumed to be representative of all four compartments of the EDC muscle, an extrinsic extensor of the finger. Both ECRL, ECRB and EDC compartments originate from the common extensor tendon attaching to the lateral epicondyle. From this common tendon, EDC spreads in four bellies with individual tendons inserting on the dorsal aspect of one of the long fingers via the extensor mechanism [22]. ECRB and ECRL both represent an individual muscle-tendon unit and inserts on the proximal part of the second metacarpal bone.

Adaptations of the protocol previously proposed [33] were required to investigate the extensors especially the tested postures were adjusted to ensure datapoints representative of the force-length portion covered by extensors $[4]$ and limiting the contribution of passive tissues [38]. Additionally, a visual guidance with feedback on EMG activity, instead of joint torque was used because voluntary producing large extension moments is rather difficult, as opposed to flexion actions which are predominant in everyday life. The EMG feedback ensured the participants were 
fully activating the targeted muscle.

\subsection{Experimental design}

\subsubsection{Participants}

Ten male participants $(24.3 \pm 5.2$ years, $177.6 \pm 7.1 \mathrm{~cm}, 70.7 \pm 6.4 \mathrm{~kg}$, hand length: $19.2 \pm$ $0.6 \mathrm{~cm}$ ) with no history of pathologies or surgeries to the right arm in the past 12 months were tested in this study. The participants gave their written informed consent in accordance with the ethics committee of Aix-Marseille University (ref: 2020-07-05-02).

\subsubsection{Tasks}

Participants performed isometric extension tasks using either the wrist or the four metacarpophalangeal (MCP) joints together in different combinations of flexion/extension angles on an ergometer specially adapted for the hand (Bio2M, Compiègne, France; Figure 1), already presented in previous studies [27, 33]. Those extension tasks consisted in progressive isometric ramps guided using a real-time feedback of the EMG activity level of the tested muscle. During those tasks, the participants were standing with the right forearm in mid-pronation, the elbow at about $120^{\circ}\left(180^{\circ}\right.$ corresponding to full extension) and the shoulder at $30^{\circ}$ of both flexion and abduction.

\subsubsection{Protocol}

Prior to the experiments, measuring tape was used to determine along with other anthropometric measurements, a reference musculotendon length ( $\left.L_{r}^{m t u}\right)$ for both muscles of each participant: from the lateral epicondyle to the metacarpal head of the index finger for EDC and from the lateral epicondyle to the base of the second metacarpal for ECR. Participants had their forearm oriented as specified as above, the fingers straight and the wrist in a neutral posture, i.e. $0^{\circ}$ of flexion and deviation.

Measurements for each participant were done in two sessions: one for the wrist extensor muscles (ECR session) and one for the finger extensor muscles (EDC session). Depending on the session, the axis of rotation of either the wrist for ECR or the metacarpophalangeal (MCP) joints for EDC was aligned with the measurement axis of the ergometer. During the ECR session, 
participants performed wrist extensions by applying forces on a $2-\mathrm{cm}$ long aluminum plate positioned at the dorsal side of the palm hand proximally to the MCP joint (Figure 1a-c). The position of the plate was such that fingers could not exert forces on it and participants were instructed to keep their fingers relaxed to reduce at best the implication of finger muscles in the moment exerted on the ergometer. During the EDC session, participants exerted forces with their fingers on a longer aluminum plate that was covering their hand from the base of the proximal phalanges to the tip of distal phalanges (Figure 1d-f).

At the beginning of each session, the participants started by a familiarization session during which they also warmed-up by applying progressively increasing isometric moments. Participants were then asked to perform two maximal voluntary contractions (MVC) in a neutral posture, i.e. wrist at $0^{\circ}$ of flexion and abduction for ECR and wrist and $\mathrm{MCP}$ at $0^{\circ}$ for EDC. If the participant expressed concerns about reaching a maximal performance or if the recorded moment was substantially different between the two trials, another trial was performed. The maximal activity $\left(\mathrm{EMG}_{\max }\right)$ of the targeted muscle (either ECR or EDC) was recorded in both MVC trials and the highest value was further used as the reference for normalization, i.e. $100 \%$. Then, the participants performed two progressive isometric ramps from low (below 5\%) to maximal activity, i.e. $100 \%$ of $\mathrm{EMG}_{\max }$ in five different joint angle configurations (described below). These ramps were guided using a real-time feedback of the participant EMG normalized activity (current and past) along with the desired ramp profile. The desired profile imposed first a "passive" period of 3 seconds where activity had to remain stable and below $5 \%$, and then an "active" period where activity had to increase linearly (ramp) during 6 seconds to reach maximal activity (100\%). During the ECR session, only the wrist posture varied, and the tested angles were $-40^{\circ},-20^{\circ}, 0^{\circ}$, $25^{\circ}, 50^{\circ}$ (negative values correspond to extension). During the EDC session, a combination of wrist $/ \mathrm{MCP}$ joint angles were tested: $-30^{\circ} / 0^{\circ}, 0^{\circ} / 0^{\circ}, 0^{\circ} / 25^{\circ}, 0^{\circ} / 50^{\circ}, 20^{\circ} / 50^{\circ}$ (wrist $/ \mathrm{MCP}$ ). These postures were carefully selected during pre-tests to limit the number of contractions, and thus minimize fatigue, while ensuring to have datapoints on both sides of the plateau region of the force-length relationship.

To test the modeled Force-Length-Activation relationships for the estimation of muscle force, 
the participants were also required to perform "sawtooth" contractions in each session. These test trials consisted of 10 seconds of isometric contraction with the participant exerting a varying moment (alternation of ascending and descending ramps) in a range from 10 to $90 \%$ of the targeted muscle activity. Two postures were tested for each session: wrist at $0^{\circ}$ and $-40^{\circ}$ for ECR and wrist $/ \mathrm{MCP}$ at $0^{\circ} / 0^{\circ}$ and $20^{\circ} / 50^{\circ}$ for EDC. These conditions were also guided via real-time feedback on activation level and were repeated twice for each posture. Only two postures were tested in order to limit the number of contractions and prevent any effect of fatigue.

All contractions (ramp and sawtooth) were randomized and participants respected a minimum of 2-minute rest before starting each trial. Verbal encouragement was given during all MVC and ramp trials to prevent at best the occurrence of submaximal performances $[39,40]$.

\subsubsection{Data acquisition and pre-processing}

A five camera motion analysis system (Vicon MX Cameras, Oxford, UK) was used to record at $100 \mathrm{~Hz}$ the three-dimensional coordinates of reflective markers placed on i) the ergometer (3 markers) to track the position of the hand, ii) the forearm (radial styloid, lateral epicondyle and biceps tendon insertion) to track the position of the radius iii) the ultrasound probe ( 3 markers) to track its orientation relative to the forearm. The biceps insertion marker was used to measure the wrist flexion/extension angle in combination with those on the ergometer and the lateral epicondyle marker was used as an estimation of the position of the origin for both muscles. The torque $(\tau)$ measured by the ergometer was recorded at $2000 \mathrm{~Hz}$ using a 12-bit analog-to-digital converter connected to the motion capture system (MX-Giganet, Vicon, Oxford, UK). EMG signals from ECR and EDC were collected at $2000 \mathrm{~Hz}$ using wireless electrodes (Biopac, MP150, Goleta, CA). The electrode placement was determined using anatomical descriptions, palpations as well as display of the signals during functional movements [27]. An ultrasound scanner (Echo Blaster 128, TELEMED, Lithuania) was used to observe the myotendinous junction with a 60 $\mathrm{Hz}$ sampling frequency. Depending on the session, the probe $(10 \mathrm{MHz}, 60 \mathrm{~mm})$ was placed on the myotendinous junction of either the ECRB or the index finger EDC compartment (EDCI). The placement of the probe was verified before beginning each trial by visualizing the ultrasound 
image in real-time while the participant executed individual finger flexion/extension and wrist flexion/extension movements. During the trial, the experimenter firmly maintained the probe on the skin while continuously tracking the myotendinous junction using the real-time feedback. The markers on the probe, representing the observation plane, were further used to reconstruct the three-dimensional position of the junction from the 2D images. During all trials, ultrasound, electromyographic (EMG) and kinematic data were collected synchronously.

The recorded torque ( $\tau$ ) was low-pass filtered at $5 \mathrm{~Hz}$ using a 2 nd order Butterworth zerophase filter. EMG envelopes were obtained by using first a band-pass filter at 10-400 Hz, rectifying and then applying low-pass filtered at $3 \mathrm{~Hz}$ using a 4th order Butterworth zero-phase filter. Those EMG envelopes were then normalized using maximum EMG envelope value obtained in the two MVC trials $\left(\mathrm{EMG}_{\max }\right)$ to calculate the muscle activation level $\left(a^{m}\right)$. Kinematic data were low-pass filtered at $5 \mathrm{~Hz}$ using a 4th order Butterworth zero-phase filter to calculate flexion/extension angles at the wrist $\left(\theta_{w}\right)$ and and $\operatorname{MCP}\left(\theta_{m c p}\right)$ joints. Only the data corresponding to the active part of the contraction was kept for further processing. For each trial, the beginning of the contraction was identified when the torque signal exceeded a threshold on the initial 3-second resting period determined as 3 standard deviations relative to its mean value, both calculated on that same period. The end of the active part was determined as the sample where the maximal envelope of the targeted muscle was reached. The mean value on the initial 3-second period was removed from the torque signal to minimize the influence of passive contributions in the analysis of force-length behaviour. To limit the number of times the musculoskeletal model was ran for each trial, the torque $\tau$, EMG, kinematic and ultrasound data were then resampled to obtain 30 and 60 samples for ramp and sawtooth contraction trials, respectively. All processing was done using Matlab (The Mathworks, Natick, USA).

\subsection{Process to obtain Force-Length-Activation relationships}

The steps to derive the Force-Length-Activation relationship of each muscle group for a single

Place Fig2 around here participant based on the processed experimental data is described in this section and illustrated on Figure 2. 


\subsubsection{Muscle force and belly length}

The displacement of the myotendinous junction was tracked manually on the B-mode image and its $3 \mathrm{D}$ coordinates were reconstructed using the position of the markers on the ultrasound probe. The muscle belly length $\left(L^{m}\right)$ was then estimated from the distance between the $3 \mathrm{D}$ coordinates of the myotendinous junction and the lateral humeral epicondyle (i.e. muscle origin). The belly length change $\left(\Delta L^{m}\right)$ during the contraction was computed as follow:

$\Delta L^{m}=L^{m}-L_{1}^{m}$

Eq. 1

where $L_{1}^{m}$ is the muscle length at rest for the current posture, i.e. at the beginning of the contraction.

The muscle force $\left(F^{m}\right)$ generated by the muscle, i.e. EDC or ECR, was estimated as in [33] using a modified version of a previously developed hand musculoskeletal model [1]. The use of such model was required to identify the individual contribution of the targeted muscles in the exertion of the net joint moment measured on the ergometer $(\tau)$. Briefly, this model used an inverse-dynamics approach and solved the muscle redundancy problem by minimizing a musclestress criterion [41] to estimate all the muscle forces required to balance the net joint moment $\tau$ in the current posture $\left(\theta_{m c p}\right.$ and $\left.\theta_{w}\right)$. The potential contribution of each muscle was quantified by two factors: its moment arm and its physiological cross-sectional area (PCSA). Moment arm values were estimated from joint angles using polynomial regression [42] and geometrical models [22]. PCSA were taken from data reported in the literature [22, 43]. For the ECR session, the model estimated the forces of the 42 hand muscles, including six wrist actuators, nine thumb muscles and 27 long finger muscles, using the wrist net moment and the wrist joint angle $\left(\theta_{w}\right)$ as input while imposing a null moment at the finger joints. The sum of ECRL and ECRB forces was used in further processing. For the EDC session, the model estimated the forces of the 27 extrinsic and intrinsic muscles crossing the MCP joints of the four long fingers using the resultant moment generated by all MCP joints and the MCP joint angle $\left(\theta_{m c p}\right)$ as input. The sum of the four EDC forces was used in further processing. For further details on those calculations, please consult the 
digital content in [33] and the study presenting the model [1].

For both muscles (EDC, ECR), the instantaneous muscle-tendon unit length ( $L^{m t u}$ ) was estimated as the sum of the measured reference one ( $L_{r}^{\text {mtu }}$ ) and the excursion, calculated from the $\operatorname{MCP}\left(\theta_{m c p}\right)$ and/or wrist joint angle $\left(\theta_{w}\right)$ using geometric models $[22,42]$.

\subsubsection{Relationships describing muscle behaviour during ramp trials}

For each ramp trial, two relationships were fitted to the data to be able to predict muscle force and belly length at specific activation levels. First, normalized force and muscle length change ( $f^{m}, \Delta l^{m}$ ) were obtained by dividing $F^{m}$ and $\Delta L^{m}$ values by their maximal value in each ramp trial. Then, a force-activation and a length-force relationship describing the muscle behaviour were obtained by fitting the equations

$$
f^{m}\left(a^{m}, \boldsymbol{\alpha}\right)=\alpha_{1}\left[\frac{1}{1+\mathrm{e}^{-\alpha_{2}\left(a^{m}-\alpha_{3}\right)}}-0.5\right]+\alpha_{4},
$$

and

$$
\Delta l^{m}\left(f^{m}, \boldsymbol{\gamma}\right)=\gamma_{1}\left[1-\mathrm{e}^{-\gamma_{2} f^{m}}\right]
$$

where $\boldsymbol{\alpha}=\left[\alpha_{1}, \alpha_{2}, \alpha_{3}, \alpha_{4}\right]$ and $\boldsymbol{\gamma}=\left[\gamma_{1}, \gamma_{2}\right]$ are constants which were determined using two successive non-linear least square fitting. The minimized criteria were

$$
, G(\boldsymbol{\alpha})=\sum_{t}\left[f^{m}(t)-f^{m}\left(a^{m}(t), \boldsymbol{\alpha}\right)\right]^{2}
$$

and

$$
H(\gamma)=\sum_{t}\left[\Delta l^{m}(t)-\Delta l^{m}\left(f^{m}(t), \gamma\right)\right]^{2}
$$

where $t$ corresponds to a time sample. Additional boundary conditions $0 \leq \alpha_{1} \leq 1 ; 0 \leq \alpha_{2} \leq 1 ; 1 \leq \alpha_{3} \leq 10 ; 0.1 \leq \alpha_{4} \leq 10$ and $\left.1 \leq \gamma_{1} \leq 10 ; 1 \leq \gamma_{2} \leq 10\right)$ and specific constraints $\left(f^{m}(0, \boldsymbol{\alpha})=0 ; f^{m}(1, \boldsymbol{\alpha})=1 ; \Delta l^{m}(0, \boldsymbol{\gamma})=0\right)$ were used to ensure physiologically realistic results [33]. The obtained relationships (Eq 2 and 3) can then be used to estimate muscle force $F^{m}$ and muscle length change $\Delta L^{m}$ from any activation level. 


\subsubsection{Derivation of Force-Length-Activation relationships}

A 3D Force-Length-Activation relationship was calculated by fitting a force-length relationship at multiple activation levels using the model of Otten $[44,45]$, which defines the force-length relationship using three parameters, i.e. the maximal isometric $\left(F_{0}\right)$, the optimal length $\left(L_{0}\right)$ and the architecture index $\left(i_{a}\right)$, i.e. muscle belly to fiber length ratio, using the equation

$$
F^{m}\left(L^{m}, F_{0}, L_{0}, i_{a}\right)=F_{0} \cdot \exp \left[-\left(\frac{\left.\left(\varepsilon^{m}+1\right)^{\beta}-1\right)}{\omega}\right)^{\rho}\right]
$$

with

$$
\begin{array}{lr}
\varepsilon^{m}=\frac{\left(L^{m}-L_{0}\right)}{L_{0}}, & \text { Eq. } 6 \mathrm{~b} \\
\omega=0.35327\left(1-i_{a}\right), & \text { Eq. } 6 \mathrm{c} \\
\beta=0.96343\left(1-\frac{1}{i_{a}}\right) \text { and } & \text { Eq. } 6 \mathrm{~d} \\
\rho=2 . & \text { Eq. } 6 \mathrm{e}
\end{array}
$$

where $\underline{\varepsilon^{m}}$ is the muscle belly strain and $\underline{\omega}, \underline{\beta}$ and $\underline{\rho}$ are parameters affecting the width, skewness and roundness of the curve, respectively. This relationship was fitted for $a^{m}$ going from $5 \%$ to $100 \%$ by steps of $5 \%$ (20 values) using an optimization process that determined the parameters $F_{0}, L_{0}, i_{a}$, for each activation level, by minimizing

$$
N\left(L_{0}, F_{0}, i_{a}\right)=\sum_{p=1}^{5}\left(F^{m}-F^{m}\left(L^{m}, F_{0}, L_{0}, i_{a}\right)\right)^{2}
$$

where using $p$ corresponds to a joint posture, defined by $\theta_{w}$ for ECR or $\theta_{m c p}$ and $\theta_{w}$ for EDC. For each $a^{m}$ value, $F^{m}$ was determined using Eq. 3 and $L^{m}$ using Eq. 4 and the resting length $L_{1}^{m}$ of the trial. Values of $F_{0}, L_{0}, i_{a}$ were constrained to remain in physiological ranges:

$0.8 \max F^{m} \leq F_{0} \leq 1.2 \max F^{m}$ Eq. $8 \mathrm{a}$ 


$$
0.95 \min L^{m} \leq L_{0} \leq 1.05 \max L^{m}
$$

$0.01 \leq i_{a} \leq 0.7$

The constraints regarding $F_{0}$ and $L_{0}$ were used to allow the process to find a maximal force and an optimal length outside the values observed in the trials and those of $i_{a}$ corresponded to data reported in the literature $[46,47]$.

To ensure a fit of good quality between the experimental data and the obtained Force-LengthActivation relationships, only the data from five ramp trials, one per posture, were used in the optimization process. Those five trials were found by running the optimization (Eq 7 and $8 \mathrm{a}-\mathrm{c}$ ) with all possible combinations of trials and keeping only the repetitions that provided the best fit, i.e. highest average $\mathrm{R}^{2}$ value across all activation levels.

Finally, the relationships between $F_{0}, L_{0}, i_{a}$ and $a^{m}$ were obtained using polynomial regressions of order 5, 3 and 3, respectively, on the 20 values of each parameter corresponding to the 20 activation levels. The orders of those regressions were carefully chosen to ensure good fit while using the same orders for all participants. The three relationships defined a participantspecific Force-Length-Activation relationship.

In order to predict the passive stretching of the muscle-tendon unit caused by different postures (section 2.3), a relationship describing the resting belly length $L_{1}^{m}$ as a function of muscle-tendon length ( $L^{m t u}$ ) was also derived from the data. It was obtained using a second order polynomial regression on the data from the same five ramp trials as those used in the derivation of Force-Length Activation relationships:

$$
L_{1}^{m}\left(L^{m t u}, \boldsymbol{d}\right)=d_{2}\left(L^{m t t u}\right)^{2}+d_{1} L^{m t u}+d_{0} .
$$

\subsubsection{Calculation of sample population relationships}

To evaluate the effect of using relationships representing the tested sample population, instead of participant-specific ones, an average Force-Length-Activation relationship was derived by applying the same regressions as for each participant on the gathered $F_{0}, L_{0}, i_{a}$ data of all 
participants for the $20 \quad a^{m}$ values. In the same way, average $f^{m}\left(a^{m}, \boldsymbol{\alpha}\right), \Delta l^{m}\left(f^{m}, \boldsymbol{\gamma}\right), L_{1}^{m}\left(L^{m t u}, \boldsymbol{d}\right)$ relationships (Eq. 3, 4 and 9, respectively) were calculated using the gathered data from the entire population. Prior to the calculation of the average model, $F^{m}, L^{m}$ and $L^{m t u}$ scaled according to the maximal isometric force ( $F_{0}^{\max }$ i.e. $F_{0}$ value for $\left.a^{m}=1\right)$, the reference muscle length, i.e. at rest in neutral posture $\left(L_{r}^{m}\right)$, and the reference musculotendon length ( $L_{r}^{m t u}$ ), respectively.

\subsection{Comparison of models based on Force-Length-Activation relationships}

Once the Force-Length-Activation relationships were obtained, their ability to evaluate

Place Fig3 around here

muscle forces was tested using the data acquired during the sawtooth contractions (Figure 3a).

Different EMG-driven models were thus developed based on the Force-Length-Activation relationships to estimate muscle forces using using kinematic $\left(\theta_{m c p}\right.$ and $\left.\theta_{w}\right)$ and $\operatorname{EMG}\left(a^{m}\right)$ data, i.e. forward dynamics approach (Figure 3b). The muscle forces estimated by those EMG-driven models were then compared to those estimated by the hand musculoskeletal model using the torque $(\tau)$ and angle data, i.e. inverse dynamics approach.

The process to evaluate muscle forces using the EMG-driven model is the same as in in Hauraix et al. [33] and is illustrated on Figure 3b. First, the resting belly length $L_{1}^{m}$ in the current posture was deduced from the muscle-tendon length (Eq. 9) which was obtained from joint angle (section 2.2.1). Then, the muscle belly length change $\Delta L^{m}$ was estimated from the activation level $a^{m}$ using Eq. 2 and 3 successively. Muscle belly length $L^{m}$ was then estimated by adding the muscle length change $\Delta L^{m}$ to the length at rest $L_{1}^{m}$. The Force-Length-Activation relationship was then determined by calculating the three parameters $\left(F_{0}, L_{0}, i_{a}\right)$ according to activation level $a^{m}$. Muscle force $F^{m}$ could then be estimated from $L^{m}$ using Eq.8a-e.

For each participant and each muscle group, three different versions of the EMG-driven model were tested: i) using the individual relationship determined for that participant (IND model), ii) using the average relationship of the sample population (AVE model) and iii) using only the 
average activation-dependent maximal isometric force of the average model $\left(F^{m}=F_{0}\left(a^{m}\right)\right)$ i.e. neglecting force-length behavior (NoFL model). The muscle force in the AVE and NoFL models was obtained by multiplying the normalized force to the maximal isometric force $F_{0}^{\max }$ of the participant. The accuracy of each model was evaluated by computing the root mean square error (RMSE) between the predicted muscle forces by that model and those obtained using the hand musculoskeletal model. The RMSE values were normalized with respect to $F_{0}^{\max }$ of the participant.

\subsection{Statistical analysis}

Normality of the data was confirmed using the Shapiro-Wilk test. R software was used to perform parametric statistical tests. The statistical differences between both muscles were tested using paired t-tests to compare the maximal net joint moment ( $\tau^{\max }$; highest value recorded with the ergometer about the wrist for ECR and MCP for EDC), maximal muscle force ( $\left.F_{0}^{\max }\right)$ muscle optimal length ( $L_{\text {opt }}$, i.e. $L_{0}$ for $\left.a^{m}=1\right)$ and architectural properties $\left(L_{r}^{m t u}, L_{r}^{m}\right.$ and $i_{a}^{o p t}$ i.e. $i_{a}$ for $a^{m}=1$ ). Two repeated-measures ANOVA (one for each muscle) were performed to assess the effect of posture ( 0 and -40 for ECR, 0/0 and 20/50 for EDC) and model (IND, AVE, or NoFL) on the RMSE of predicted muscle forces. A Tukey post-hoc analysis was conducted when appropriate. The level of significance was set to $p<0.05$.

\section{Results}

\subsection{Architectural data}

Table 1 summarizes the measured and estimated architectural characteristics of EDC and ECR

Place Table1 around here muscles. Significant differences were found between the ECR and the EDC muscles $(p<0.01)$ for the maximal measured net joint moment $\left(\tau^{\max }\right)$, the muscle maximal force $\left(F_{0}^{\max }\right)$ as well as for the reference muscle-tendon length ( $\left.L_{r}^{m t u}\right)$, the reference muscle belly length at rest $\left(L_{r}^{m}\right)$, the optimal length $\left(L_{o p t}\right)$ and the optimal length normalized by $L_{r}^{m}\left(l_{\text {opt }}\right)$. Only the index of 
architecture at optimal length $\left(i_{a}^{\text {opt }}\right)$ was not significantly different between the two muscles $(p=0.89)$.

The parameters estimated and measured in the current study are in the range of data reported for ECRL and ECRB and for the four compartments of EDC in anatomical dataset acquired during dissections (Figure 4). For ECR, the architectural parameters, i.e. belly and fiber length and index of architecture, were closer to those reported for ECRB than for ECRL. For EDC, the estimated architectural data agreed with the range of values reported in the literature for the four compartments. The comparison with a specific compartment of EDC was not possible considering the large variations observed between different studies, e.g. optimal belly length ranging from 10 to $27 \mathrm{~cm}$. Compared to estimates based on PCSA from cadaver specimens, the maximal isometric force obtained for ECR $(498 \pm 115 \mathrm{~N})$, was four times higher than the lowest value, i.e. $119,5 \mathrm{~N}$ using [46], but comparable to the highest value, i.e. $429 \mathrm{~N}$ in [19]. Estimated maximal isometric force of EDC group $(169 \pm 29.3 \mathrm{~N})$, was in the range of estimates based on PCSA, i.e. from $79.9 \mathrm{~N}[47]$ to $307.2 \mathrm{~N}[19]$.

\subsection{Modelled Force-length-activation relationships}

Place Fig5 around here
Place Fig6 around here
The individual Force-Length-Activation relationships fitted for each participant using Eq. 8 presented a mean $\mathrm{R}^{2}$ value of $0.84 \pm 0.12$ and $0.83 \pm 0.2$ for ECR and EDC, respectively. The average relationships across the population are showed Figure 5.

Figure 6 presents the evolution the three parameters $\left(F_{0}, L_{0}, i_{a}\right)$ describing the Force-LengthActivation relationships as a function of activation level. Coefficients for the polynomial regression describing the average curves are provided in Online Resource 1. The index of architecture $\left(i_{a}\right)$ tended to increase with activation. It reached around 0.3 at maximal activation for both muscles but was slightly higher for ECR, indicating a wider force-length curve, at low activation (see Figure 5). The muscle optimal belly length $\left(L_{0}\right)$ decreased with activation for ECR, from $0.97 L_{r}^{m}$ to $0.90 L_{r}^{m}$, and remained stable for EDC, around 1 on average. The isometric maximal force $\left(F_{0}\right)$ values increased non-linearly for both muscles. 
Place Fig7 around here

\subsection{Comparison of different Force-Length-Activation models}

For both muscles, the muscle forces estimated by the three EMG-driven models (i.e. IND, AVE, NoFL) were comparable to those of the inverse-dynamics model (Figure 7, upper panels). For ECR, the RMSE was statistically different between the three models $(\mathrm{F}(2,18)=3.77 ; p=0.029)$ but not between the neutral and extension postures $(\mathrm{p}=0.16)$. No interaction effect was found. The post-hoc analysis showed that the IND model provided the lowest RMSE $(\mathrm{p}<0.05)$ and that the AVE and NoFL were not statistically different $(\mathrm{p}=0.99)$. For EDC, the RMSE was not different across models $(\mathrm{F}(2,16)=2.29 ; p=0.11)$ nor between the two postures $(\mathrm{F}(2,16)=0.54 ; p=0.47)$.

The muscle strain $\underline{\varepsilon^{m}}$ (Eq. 6b) were different between the two postures except for the NoFL model, which assumes the muscle is always at optimal length (Figure 7, centre panels). The maximal moment applied by the subjects during the sawtooth conditions were similar between the two postures (Figure 7, lower panels).

\section{Discussion}

The objective of this study was to model the Force-Length-Activation behaviour of hand extensor muscles by using non-invasive methods. The methodology developed for flexors [33] was modified to provide new insight into the muscle mechanics of finger and wrist extensors which plays a crucial role in the control of grasping [3]. To investigate the specific anatomy of the hand, two muscle groups were studied with ECR representing extensors acting only at the wrist and EDC representing extensors acting both at the finger and the wrist joints. Using a combination of experimental measurements during isometric force-varying tasks together with musculoskeletal modelling, a three-dimensional Force-Length-Activation relationship was obtained for each muscle group. Compared to the protocol for flexors, the tested postures were adjusted to match the range of action of extensors and the visual feedback guiding participants during the isometric torque exertions was based on activation levels, instead of joint torque. To our knowledge, this study is the first to provide a model considering the activation-dependency of the force-length relationships for both finger and wrist extensors which were derived from in vivo data describing the actual performances and muscle behaviour of participants. The obtained 
Force-Length-Activation relationships highlighted how hand posture differently affects the mechanics of finger muscles and wrist actuators. The results of the EMG-driven models also showed that muscle forces estimated from kinematic and EMG using those relationships were comparable to those of an inverse-dynamics musculoskeletal model.

\subsection{Architectural properties and maximal isometric force}

Compared to EDC, the results showed that ECR presented on average a twice higher force capacity (498N against $170 \mathrm{~N})$, comparable index of architecture (around 0.3 ) as well as a shorter optimal length $(14.6 \mathrm{~cm}$ against $20.9 \mathrm{~cm})$ that was further from the neutral posture $\left(0.91 L_{r}^{m}\right.$ against $0.99 L_{r}^{m}$ ). Those values estimated via the combination of in vivo measurements and musculoskeletal modeling were in agreement with direct measurements from dissection studies $[19-21,46,47]$ (Table 1 and Figure 4).

Compared to values reported for ECRB and ECRL muscles, the architectural parameters, i.e. $i_{a}^{\text {opt }}$ and $L_{\text {opt }}$, estimated for the ECR group agreed well with those reported for ECRB and especially with results of Lieber et al. $[46,47]$. This observation is consistent with the fact that, despite ECRL and ECRB were modelled as a group, the muscle shortening for ECR was interpreted from the tracking of ECRB myotendinous junction. Hence despite a limited amount of imaging data, the methodology proposed here resulted in physiologically relevant architecture. Unfortunately, comparison between the EDC group and EDCI, tracked here, was difficult because of the spread of values reported in the literature. The EDCI muscle belly length particularly exhibited a large range, i.e. from $10.4 \mathrm{~cm}[20]$ to $26.9 \mathrm{~cm}[21]$. Although the anthropometry of the specimen could be a reason for such large variations, Kerkhof et al. [21] found that dimensions of the forearm, such as radius length, do not seem to correlate with those of the muscle-tendon units. Despite those variations, the estimated parameters for EDC agree with the range reported for the four compartments and the standard deviations also demonstrate higher variation for muscle belly length $(3.9 \mathrm{~cm})$ than for other parameters.

Maximal isometric force $F_{0}^{\max }$ derived from the approach presented here also agreed well 
with estimations based on PCSA data from dissection studies (Table 1 and Figure 4). The estimated value of ECR was globally higher than estimates from PCSA while the one of EDC was in the range of the literature. This result is in accordance with previous works from Goislard de Monsabert at al. [27]. They found that the discrepancies between hand muscle capacities estimated from cadaver-based data, including PCSA, and from dynamometry were higher for wrist extensors, including ECRB and ECRL, than for finger extensors, including EDC. However, the fact that the EDC maximal isometric force estimated based on effective strength measurements was lower than when using cadaver PCSA of [19] and [21] was surprising. The results from Goislard de Monsabert at al. [27] indeed indicated that muscle capacities of finger extensors derived from dynamometry were twice higher than with PCSA taken from Chao et al.[22]. This probably indicates that discrepancies between estimates from dynamometry and from cadaver data depends on the specimens from which anatomical data were measured. Nevertheless, as few information about the specimens are available, the reasons explaining the discrepancies between the different datasets remain unclear.

Overall, the results on both architectural parameters and maximal isometric force confirm previous findings of the literature that hand musculature is variable. Variations have been observed at the anatomical level, e.g. supplementary or fusion of muscle bellies in EDC [48], and at the level of muscle group capacities with imbalances that might result from personal factors, such as expertise or pathology $[27,37]$. The development of larger anatomical datasets as well as scaling procedures based on in vivo measurements appears necessary to allow representing the muscle capacities of specific populations. The present methodology could also be improved to provide a finer understanding of the hand muscle capacities, for instance by characterizing intrinsic muscles, including radial/ulnar deviation torque measurements, or velocity-dependent aspects.

\subsection{Force-Length-Activation relationships}

The Force-Length-Activation relationships obtained here allowed to study the effect of activation on the parameters describing how length affects muscle force, namely muscle optimal 
length $\left(L_{0}\right)$, maximal isometric force $\left(F_{0}\right)$ and index of architecture $\left(i_{a}\right)$ (Figure 5 and 6$)$. The approach based on isometric ramp tasks and using a modelling approach allowed assessing the force-length relationships of hand extensors and how activation can modify the shape and position of these relationships. Using the equation of Otten [44] (Eq. 6a), the derived models provided a good interpretation of the activation- and length-dependency of muscle force with $\mathrm{R}^{2}$ values that were above 0.8 for both muscles, i.e. $0.84 \pm 0.12$ and $0.83 \pm 0.2$ across all participants for ECR and EDC, respectively. For a better comparison with the literature and with previous work from our group, Figure 8 presents the Force-Length-Activation relationships of the two extensors along with those of the two flexors previously studied [33] and highlights the portion covered over the tested postures tested as well as the effects of activation.

The portions of the force-length relationship found in the present study agreed well with those already observed through in vivo sarcomere length measurements of ECRB and ECRL $[4,6]$. The estimated Force-Length-Activation relationship indicated that ECR mainly worked on the plateau and descending limb of the force-length relationships. The muscle reached its maximal capacity in a slightly extended wrist, near $20^{\circ}$, which was closer to neutral compared to what sarcomere length measurements predicted, i.e. $40-50^{\circ}[4,6]$. This slight difference might arise from the different scales at which the force-length behaviour was studied. Considering the non-uniform distribution of sarcomeres and the non-homogeneous mechanical properties of the different tissues within a muscle [49], a complete agreement between the present study at the muscle level and sarcomere behavior was not expected. As no data was available in the literature regarding the force-length behavior of EDC compartments, no comparison can be made regarding the present results. Nevertheless, the good agreement regarding the portions of the curve covered by ECR over the range of tested postures tend to validate the use of myotendinous junction displacement, dynamometry, and electromyography to study force-length relationship at the muscle level.

The obtained Force-Length-Activation relationships provide a better understanding of how the capacities of wrist and finger extensors are affected by hand posture during everyday life. The tested postures indeed explored most of the functional ranges of motion required for everyday life 
activities, i.e. from $40^{\circ}$ extension to $40^{\circ}$ flexion at the wrist $[50]$ and between neutral to $50^{\circ}$ flexion for MCP joints [51]. In comparison with ECR, EDC is affected by both wrist and finger posture but was exposed to lesser loss of force capacities among the tested postures (Figure 8). EDC remained on the plateau region when moving only the fingers, but the effect of wrist posture was substantial as the additional lengthening or shortening resulted in a loss of more than $10 \%$ of its maximal capacities. Nevertheless, when considering the maximal activation, EDC only lost $20 \%$ of its maximal capacity in the most extreme postures whereas ECR lost around $60 \%$, near $50^{\circ}$ flexion. Although the range of motion of EDC is more complex to study because of the multiple joints affecting its length, this result suggests EDC muscle capacity is less affected by changes in length than ECR over functional ranges of motion.

Regarding the configuration corresponding to optimal length, EDC reached its maximal force for slightly flexed MCP joints, around $25^{\circ}$, with the wrist in neutral posture. Interestingly, as EDC muscle length is affected by both finger joints and the wrist, other hand postures could place this muscle at optimal length. For instance, from the posture mentioned above, a more flexed MCP joint, causing a lengthening, combined to an extended wrist, causing a shortening, would also place EDC at optimal length. Since ECR would also be close to optimal capacities in that posture, such mechanism could explain why the highest maximal grip force are reached for slightly extended wrist posture, i.e. around 25 degrees [7]. During grasping tasks, fingers are indeed flexed to maintain the object and while a wide range of wrist posture could be taken, a wrist extension could place both EDC and ECR at their optimal length. As both this muscle can balance the flexion moments created by extrinsic flexors [2,3], the wrist extension might represent an optimal posture that place both extensors in a more advantageous configuration. In another wrist posture, the extensors capacities might be significantly reduced which might prevent their ability to balance finger flexor actions at the wrist, therefore limiting the maximal grip force. Considering the crucial role of finger and wrist extensors during grasping tasks, further studies could investigate how wrist posture might modulate the coordination between hand flexors and extensors and further affect grip performance. 


\subsection{Activation-dependency of the force-length relationship}

As it has been observed for many years [34,36], a shift of optimal belly length $L_{0}$ toward longer length with decreasing activation was found with the obtained Force-Length-Activation relationships (Figure 4, 6 and 8). This shift reached 7.5\% for ECR and 3\% for EDC for null activation which remained among the lowest value reported in the literature for human muscles, i.e. from 0 [52] to $40 \%$ [53], and used in musculoskeletal models, i.e. $15 \%[54,55]$. The results from this study thus suggest the amount of this shift varies for different muscles. This result cannot be fully explained as the exact reason for this shift remains unclear. Although it has often been attributed to a length-dependence of calcium sensitivity $[35,36,56]$, it might also be caused by the force transmission mechanisms within the muscle-tendon unit [34]. This second hypothesis suggests the fibre at which the maximal capacity is reached might change with the absolute force level to optimize the efficacy of the transmission within the complex arrangement of both active and passive tissues [57]. This might explain why different optimal length shift were observed for both extensors as their structural arrangements differ. ECRB presents specific fibre trajectories with an L-shaped belly while EDC is multi-compartment muscles which are interconnected by passive tissues $[48,58]$. This hypothesis that muscle-tendon unit structural arrangement would influence activation-dependency of the force-length relationship seems confirmed by differences found in our previous work on flexors (Figure 8, FDS and FCR). Finger (FDS) and wrist (FCR) flexors indeed show similar anatomical differences than extensors, multi-compartment vs single belly muscles, and are differently affected by activation.

The shape of the force-length relationship curve, influenced by $i_{a}$, was also influenced by the activation (Figure 4, 6 and 8). The curve of ECR became flatter for activation below 0.5 while the one of EDC conserved a similar width from 1 to 0.25 . This result indicate ECR forcegenerating capacities are less affected by muscle length at low activation than EDC. This comparison of wrist actuators and finger muscles result in a different trend for flexors, with the digital flexors (FDS) being less affected by muscle length than wrist flexors (FCR). As discussed above, the exact reasons for such differences in the activation-dependency of the hand muscles 
remain unclear, although it could be the result of high specialization of each muscle as already demonstrated from architectural measurements $[46,47]$.

Although the significance of the activation-dependency might vary among hand muscles, e.g. low shift of optimal length for EDC, it is visible from the comparison of different hand muscles that they each possess unique muscle mechanics (Figure 8). The relationships obtained here (and available from Online Resource 1) allow considering the non-linearities introduced by both activation and muscle length in the estimation of finger and wrist muscle forces. Such data might clarify how hand joint configuration could affect the force-generating capacities of each muscle group and in turn influence muscle coordination.

\subsection{Results from the EMG-driven models}

The muscle forces estimated by the different EMG-driven models based on the obtained Force-Length-Activation relationships were slightly different, below $15 \%$ of $F_{0}^{\max }$ average, compared to those of the musculoskeletal model which used an inverse dynamics approach (Figure 7). Although some statistical differences were found for ECR, the models based on the participant-specific (IND) relationships resulted in similar errors compared to the sample population (AVE) model. This result corroborates the conclusion of our first study on hand flexors [33] that an average model provides a good representation that should be applicable to other participants as long as they remain in the range of age and anthropometry of the sample population. Force-length characteristics might indeed vary according to personal factors, such as training or expertise [56].

Surprisingly, the comparison of EMG-driven models indicated that the NoFL model resulted in similar errors than other models for the two tested postures (Figure 7). A high difference between the different versions of the EMG-driven models was not expected for the neutral posture since the muscles are close to their optimal length in this posture (Figure 8). Nevertheless, the difference between models for the second posture (Figure 8; star markers) of sawtooth contractions was expected to be higher as it corresponds to sub-optimal length, shorter for ECR and longer for EDC. As shown on Figure 8, the muscle force estimates of the NoFL model, 
assuming the muscle stays at optimal length (circle markers) overestimate the actual force level in this second sawtooth posture (star markers). This overestimation of NoFL varies according to the activation level but reached up to $20 \%$ for ECR and $30 \%$ for EDC. Despite those clear differences between EMG-driven models, the RMSE error calculated in the second sawtooth posture were comparable between them, approximately $10 \%$ (Figure 7). This lack of difference is explained by the fact that the muscle force estimates of the inverse-dynamics model, used as reference, were in between those of the different EMG-driven models. While AVE and IND models estimated sub-optimal muscle force, nearly $10 \%$ lower than the inverse-dynamics model, the NoFL model assumed an optimal muscle force, nearly $10 \%$ higher than the inverse-dynamics model. Those discrepancies illustrate the limitations of both the forward-dynamics, here EMGdriven models, and inverse-dynamics approaches of musculoskeletal modelling. The former is based on hypotheses at the muscle level but neglects the global performance, e.g. resultant force or motion, while the latter uses this global performance and make important hypotheses at the joint level on muscle coordination [41]. Therefore, although the inverse-dynamics musculoskeletal model was taken as reference in our study, both approaches are making different assumptions on the functioning of the neuromusculoskeletal system which results in different estimations of the force of an individual muscle. Further studies should focus on the development of new biomechanical models of the hand integrating both the inverse and direct approaches based on the relationships found in the present study, as it has been done for the lower limb [54, 59]. Nevertheless, considering the relatively good fit quality with experimental data and the relatively low RMSE with the musculoskeletal model estimates, we consider that our ForceLength-Activation relationships represent, by themselves, an appropriate model of how extensors' muscle forces are dependent to both activation and length.

\subsection{Limitations}

Some limitations should be considered when interpreting the results of the present study. It has been shown that ramp contractions can underestimate the maximal muscle force as it depends on fibre shortening velocity and contraction history [60]. This underestimation, also called force depression, seems to increase linearly with the mechanical work the muscle produces during the 
contraction. To estimate this possible bias, the contraction work during the ramp trials was calculated by integrating the instantaneous product of muscle force and was found to be on average $2.0 \pm 1.2 \mathrm{~mJ} / \mathrm{N}$ and $1.4 \pm 0.9 \mathrm{~mJ} / \mathrm{N}$ for ECR and EDC, respectively, once normalized by maximal muscle force. As this estimation would correspond to force depression levels below $5 \%$ of maximal isometric force $[60,61]$, we consider the ramp trials designed for this study were slow enough to minimize this phenomenon. Another limitation concerns the use of voluntary contractions, which might not result in the "true" maximal performance [40]. Nevertheless, we consider that the precautions taken, including the EMG-based visual guidance, verbal encouragements and randomizing of ramp postures, have minimized the factors that could influence the occurrence of sub-maximal performances. The modelling approach proposed here does not allow a quantification of the fine muscle-tendon unit mechanics, such as the threedimensional behaviour of the different tissues [62] or the dynamic equilibrium between muscle and tendon strain [63]. Nevertheless, the good agreement between our estimations based on in vivo measurements and previous findings regarding muscle architecture, sarcomere length and activation-dependency tends to confirm the obtained Force-Length-Activation relationships provide an adequate model of the muscle behaviour of hand extensors.

\section{Conclusion}

This study provided a model of the force-length behaviour of the ECR and EDC muscles considered as representative of wrist and finger extensors, respectively. The procedure combining musculoskeletal model with in vivo data of joint torque performance and muscle behaviour provided new insight in the activation- and length-dependency of hand extensors. Based on the obtained Force-Length-Activation relationships, an EMG-driven model was proposed to evaluate muscle force using electromyography and joint posture and provided results in agreement with an inverse-dynamics model. Those EMG-driven models could be helpful for clinical or ergonomic applications as they are easier to implement compared to inverse-dynamics models which requires the use of numerous input data and anatomical parameters. Nevertheless, for a better accuracy, the results suggested a combination of both approaches would result in finer estimates of muscle 
forces. The data describing the Force-Length-Activation relationships provided in Online Resource 1 could thus be used by interested parties who seek to estimate the muscle capacities of hand extensors. For instance, considering that overloading of extensor muscles relative to their capacities represent a risk factor for lateral epicondylalgia [37], these relationships could help to design tool handles and recommendations for manual tasks such that extensor muscles remain close to their optimal length when they are highly involved. By describing how muscle capacities are affected by joint posture, the models obtained in the present study could also be used to clarify how biomechanical constraints might influence the muscle coordination during grasping tasks.

\section{Acknowledgements}

The authors declare that the results of the study are presented clearly, honestly, and without fabrication, falsification, or inappropriate data manipulation. There were no external funding sources used in the preparation of this article.

\section{Compliance with Ethical Standards}

There is no conflict of interests concerning the preparation of this article.

This study was performed in line with the principles of the Declaration of Helsinki. Approval was obtained from the local ethics committee of Aix-Marseille University.

\section{References}

1. Goislard de Monsabert B, Rossi J, Berton E, Vigouroux L (2012) Quantification of hand and forearm muscle forces during a maximal power grip task. Med Sci Sports Exerc 44:1906-1916. https://doi.org/10.1249/MSS.0b013e31825d9612

2. Snijders CJ, Volkers AC, Mechelse K, Vleeming A (1987) Provocation of epicondylalgia lateralis (tennis elbow) by power grip or pinching. Med Sci Sports Exerc 19:518-523

3. Charissou C, Amarantini D, Baurès R, et al (2017) Effects of hand configuration on muscle force coordination, co-contraction and concomitant intermuscular coupling during maximal isometric flexion of the fingers. Eur $J$ Appl Physiol 117:2309-2320. https://doi.org/10.1007/s00421-017-3718-6

4. Lieber RL, Loren GJ, Fridén J (1994) In vivo measurement of human wrist extensor muscle sarcomere length changes. J Neurophysiol 71:874-881. 
https://doi.org/10.1152/jn.1994.71.3.874

5. Lieber RL, Ljung BO, Fridén J (1997) Intraoperative sarcomere length measurements reveal differential design of human wrist extensor muscles. J Exp Biol 200:19-25

6. Cromie MJ, Sanchez GN, Schnitzer MJ, Delp SL (2013) Sarcomere lengths in human extensor carpi radialis brevis measured by microendoscopy: Sarcomere Lengths in Human ECRB. Muscle Nerve 48:286-292. https://doi.org/10.1002/mus.23760

7. O'Driscoll SW, Horii E, Ness R, et al (1992) The relationship between wrist position, grasp size, and grip strength. J Hand Surg 17:169-177. https://doi.org/10.1016/03635023(92)90136-D

8. Dennerlein JT, Diao E, Mote CD, Rempel DM (1998) Tensions of the flexor digitorum superficialis are higher than a current model predicts. J Biomech 31:295-301

9. An KN, Chao EY, Cooney WP, Linscheid RL (1985) Forces in the normal and abnormal hand. J Orthop Res 3:202-211. https://doi.org/10.1002/jor.1100030210

10. Goislard de Monsabert B, Vigouroux L, Bendahan D, Berton E (2014) Quantification of finger joint loadings using musculoskeletal modelling clarifies mechanical risk factors of hand osteoarthritis. Med Eng Phys 36:177-184. https://doi.org/10.1016/j.medengphy.2013.10.007

11. Mirakhorlo M, Van Beek N, Wesseling M, et al (2018) A musculoskeletal model of the hand and wrist: model definition and evaluation. Comput Methods Biomech Biomed Engin 110. https://doi.org/10.1080/10255842.2018.1490952

12. Valero-Cuevas FJ, Zajac FE, Burgar CG (1998) Large index-fingertip forces are produced by subject-independent patterns of muscle excitation. J Biomech 31:693-703. https://doi.org/10.1016/S0021-9290(98)00082-7

13. Gonzalez RV, Buchanan TS, Delp SL (1997) How muscle architecture and moment arms affect wrist flexion-extension moments. J Biomech 30:705-712

14. Nichols JA, Bednar MS, Havey RM, Murray WM (2015) Wrist salvage procedures alter moment arms of the primary wrist muscles. Clin Biomech 30:424-430. https://doi.org/10.1016/j.clinbiomech.2015.03.015

15. Sancho-Bru JL, Pérez-González A, Vergara-Monedero M, Giurintano D (2001) A 3-D dynamic model of human finger for studying free movements. J Biomech 34:1491-1500. https://doi.org/16/S0021-9290(01)00106-3

16. Loren GJ, Shoemaker SD, Burkholder TJ, et al (1996) Human wrist motors: Biomechanical design and application to tendon transfers. J Biomech 29:331-342. https://doi.org/10.1016/0021-9290(95)00055-0

17. Wohlman SJ, Murray WM (2013) Bridging the gap between cadaveric and in vivo experiments: A biomechanical model evaluating thumb-tip endpoint forces. J Biomech 46:1014-1020. https://doi.org/10.1016/j.jbiomech.2012.10.044

18. Kamper DG, Fischer HC, Cruz EG (2006) Impact of finger posture on mapping from muscle activation to joint torque. Clin Biomech 21:361-369. https://doi.org/10.1016/j.clinbiomech.2005.11.005

19. Goislard De Monsabert B, Edwards D, Shah D, Kedgley A (2018) Importance of Consistent 
Datasets in Musculoskeletal Modelling: A Study of the Hand and Wrist. Ann Biomed Eng 46:71. https://doi.org/10.1007/s10439-017-1936-z

20. Mirakhorlo M, Visser JMA, Goislard de Monsabert BAAX, et al (2016) Anatomical parameters for musculoskeletal modeling of the hand and wrist. International Biomechanics 3:40-49. https://doi.org/10.1080/23335432.2016.1191373

21. Kerkhof FD, van Leeuwen T, Vereecke EE (2018) The digital human forearm and hand. Journal of Anatomy 233:557-566. https://doi.org/10.1111/joa.12877

22. Chao EY, An KN, Cooney III WP, Linscheid RL (1989) Biomechanics of the hand: a basic research study. World Scientific, Singapore

23. Gordon AM, Huxley AF, Julian FJ (1966) The variation in isometric tension with sarcomere length in vertebrate muscle fibres. J Physiol 184:170-192

24. Holzbaur KRS, Murray WM, Delp SL (2005) A model of the upper extremity for simulating musculoskeletal surgery and analyzing neuromuscular control. Ann Biomed Eng 33:829840. https://doi.org/10.1007/s10439-005-3320-7

25. Heinen F, Lund ME, Rasmussen J, Zee M de (2016) Muscle-tendon unit scaling methods of Hill-type musculoskeletal models: An overview: Proceedings of the Institution of Mechanical Engineers, Part H: Journal of Engineering in Medicine. https://doi.org/10.1177/0954411916659894

26. Hicks JL, Uchida TK, Seth A, et al (2015) Is My Model Good Enough? Best Practices for Verification and Validation of Musculoskeletal Models and Simulations of Movement. J Biomech Eng 137:0209051-02090524. https://doi.org/10.1115/1.4029304

27. Goislard de Monsabert B, Rao G, Gay A, et al (2017) A scaling method to individualise muscle force capacities in musculoskeletal models of the hand and wrist using isometric strength measurements. Med Biol Eng Comput 55:2227-2244. https://doi.org/10.1007/s11517-017-1662-6

28. Armstrong TJ, Chaffin DB (1978) An investigation of the relationship between displacements of the finger and wrist joints and the extrinsic finger flexor tendons. $J$ Biomech 11:119-128

29. Hatze H (1981) Estimation of myodynamic parameter values from observations on isometrically contracting muscle groups. Europ J Appl Physiol 46:325-338. https://doi.org/10.1007/BF00422120

30. Leedham JS, Dowling JJ (1995) Force-length, torque-angle and EMG-joint angle relationships of the human in vivo biceps brachii. Europ J Appl Physiol 70:421-426. https://doi.org/10.1007/BF00618493

31. Maganaris CN (2003) Force-length characteristics of the in vivo human gastrocnemius muscle. Clin Anat 16:215-223. https://doi.org/10.1002/ca.10064

32. Winter SL, Challis JH (2010) The Force-Length Curves of the Human Rectus Femoris and Gastrocnemius Muscles in Vivo. J Appl Biomech 26:45-51. https://doi.org/10.1123/jab.26.1.45

33. Hauraix H, Goislard De Monsabert B, Herbaut A, et al (2018) Force-Length Relationship Modeling of Wrist and Finger Flexor Muscles. Med Sci Sports Exerc 50:2311-2321. https://doi.org/10.1249/MSS.0000000000001690 
34. Holt NC, Azizi E (2014) What drives activation-dependent shifts in the force-length curve? Biol Lett 10:20140651. https://doi.org/10.1098/rsbl.2014.0651

35. Rockenfeller R, Günther M (2018) Inter-filament spacing mediates calcium binding to troponin: A simple geometric-mechanistic model explains the shift of force-length maxima with muscle activation. Journal of Theoretical Biology 454:240-252. https://doi.org/10.1016/j.jtbi.2018.06.009

36. Rack PMH, Westbury DR (1969) The effects of length and stimulus rate on tension in the isometric cat soleus muscle. J Physiol 204:443-460. https://doi.org/10.1113/jphysiol.1969.sp008923

37. Vigouroux L, Goislard de Monsabert B, Hayot C, et al (2017) Assessment of the risk and biomechanical consequences of lateral epicondylalgia by estimating wrist and finger muscle capacities in tennis players. Sports Biomech 16:434-451. https://doi.org/10.1080/14763141.2016.1212916

38. Delp SL, Grierson AE, Buchanan TS (1996) Maximum isometric moments generated by the wrist muscles in flexion-extension and radial-ulnar deviation. J Biomech 29:1371-1375. https://doi.org/10.1016/0021-9290(96)00029-2

39. McNair PJ, Depledge J, Brettkelly M, Stanley SN (1996) Verbal encouragement: effects on maximum effort voluntary muscle: action. British Journal of Sports Medicine 30:243-245. https://doi.org/10.1136/bjsm.30.3.243

40. Gandevia SC (2001) Spinal and Supraspinal Factors in Human Muscle Fatigue. Physiological Reviews 81:1725-1789. https://doi.org/10.1152/physrev.2001.81.4.1725

41. Crowninshield RD, Brand RA (1981) A physiologically based criterion of muscle force prediction in locomotion. J Biomech 14:793-801. https://doi.org/10.1016/00219290(81)90035-X

42. Lemay MA, Crago PE (1996) A dynamic model for simulating movements of the elbow, forearm, and wrist. J Biomech 29:1319-1330. https://doi.org/16/0021-9290(96)00026-7

43. Ramsay JW, Hunter BV, Gonzalez RV (2009) Muscle moment arm and normalized moment contributions as reference data for musculoskeletal elbow and wrist joint models. J Biomech 42:463-473. https://doi.org/10.1016/j.jbiomech.2008.11.035

44. Otten E (1987) A myocybernetic model of the jaw system of the rat. J Neurosci Methods 21:287-302

45. Kaufman KR, An KN, Chao EY (1989) Incorporation of muscle architecture into the muscle length-tension relationship. J Biomech 22:943-948

46. Lieber RL, Fazeli BM, Botte MJ (1990) Architecture of selected wrist flexor and extensor muscles. J Hand Surg 15:244-250. https://doi.org/10.1016/0363-5023(90)90103-X

47. Lieber RL, Jacobson MD, Fazeli BM, et al (1992) Architecture of selected muscles of the arm and forearm: Anatomy and implications for tendon transfer. J Hand Surg 17:787-798. https://doi.org/10.1016/0363-5023(92)90444-T

48. Leijnse JNAL, Carter S, Gupta A, McCabe S (2008) Anatomic Basis for Individuated Surface EMG and Homogeneous Electrostimulation With Neuroprostheses of the Extensor Digitorum Communis. Journal of Neurophysiology 100:64-75. https://doi.org/10.1152/jn.00706.2007 
49. Moo EK, Leonard TR, Herzog W (2020) The sarcomere force-length relationship in an intact muscle-tendon unit. J Exp Biol 223:jeb215020. https://doi.org/10.1242/jeb.215020

50. Ryu J, Cooney WP, Askew LJ, et al (1991) Functional ranges of motion of the wrist joint. J Hand Surg 16:409-419. https://doi.org/10.1016/0363-5023(91)90006-W

51. Roda-Sales A, Vergara M, Sancho-Bru JL, et al (2019) Human hand kinematic data during feeding and cooking tasks. Sci Data 6:167. https://doi.org/10.1038/s41597-019-0175-6

52. de Brito Fontana H, Herzog W (2016) Vastus lateralis maximum force-generating potential occurs at optimal fascicle length regardless of activation level. Eur J Appl Physiol 116:1267-1277. https://doi.org/10.1007/s00421-016-3381-3

53. Ichinose Y, Kawakami Y, Ito M, Fukunaga T (1997) Estimation of active force-length characteristics of human vastus lateralis muscle. Acta Anat 159:78-83. https://doi.org/10.1159/000147969

54. Lloyd DG, Besier TF (2003) An EMG-driven musculoskeletal model to estimate muscle forces and knee joint moments in vivo. $J$ Biomech 36:765-776. https://doi.org/10.1016/S0021-9290(03)00010-1

55. Cox SM, Easton KL, Lear MC, et al (2019) The Interaction of Compliance and Activation on the Force-Length Operating Range and Force Generating Capacity of Skeletal Muscle: A Computational Study using a Guinea Fowl Musculoskeletal Model. Integrative Organismal Biology 1:obz022. https://doi.org/10.1093/iob/obz022

56. Rassier DE, MacIntosh BR, Herzog W (1999) Length dependence of active force production in skeletal muscle. J Appl Physiol 86:1445-1457

57. Huijing PA (1999) Muscle as a collagen fiber reinforced composite: a review of force transmission in muscle and whole limb. $J$ Biomech 32:329-345. https://doi.org/10.1016/S0021-9290(98)00186-9

58. von Schroeder HP, Botte MJ (1993) The functional significance of the long extensors and juncturae tendinum in finger extension. J Hand Surg Am 18:641-647. https://doi.org/10.1016/0363-5023(93)90309-Q

59. Amarantini D, Rao G, Berton E (2010) A two-step EMG-and-optimization process to estimate muscle force during dynamic movement. J Biomech 43:1827-1830. https://doi.org/10.1016/j.jbiomech.2010.02.025

60. Siebert T, Rode C, Herzog W, et al (2008) Nonlinearities make a difference: comparison of two common Hill-type models with real muscle. Biol Cybern 98:133-143. https://doi.org/10.1007/s00422-007-0197-6

61. Corr DT, Herzog W (2016) A cross-bridge based model of force depression: Can a single modification address both transient and steady-state behaviors? Journal of Biomechanics 49:726-734. https://doi.org/10.1016/j.jbiomech.2016.02.005

62. Seydewitz R, Siebert T, Böl M (2019) On a three-dimensional constitutive model for history effects in skeletal muscles. Biomech Model Mechanobiol 18:1665-1681. https://doi.org/10.1007/s10237-019-01167-9

63. Millard M, Uchida T, Seth A, Delp SL (2013) Flexing Computational Muscle: Modeling and Simulation of Musculotendon Dynamics. J Biomech Eng 135:021005. https://doi.org/10.1115/1.4023390 
64. Walker SM, Schrodt GR (1974) I segment lengths and thin filament periods in skeletal muscle fibers of the Rhesus monkey and the human. Anat Rec (Hoboken) 178:63-81 


\section{Figure legends}

Figure 1 - Photographs illustrating the position of the hand in the ergometer and the different plates on which participants exerted extension moments for the ECR (panels a, b and c) and EDC sessions (d, e and f). For the ECR session (a), the participants aligned the wrist joint with the measurement axis and applied the extension moment using the dorsal aspect of the hand palm. The hand position for the 0 and the $50^{\circ}$ postures of the ECR session are illustrated on panels $b$ and c, respectively. For the EDC session (d), the participants aligned their MCP joints with the measurement axis and applied the extension moment using the dorsal aspect of the fingers. The hand position for the $0 / 0$ and the $0 / 50$ (Wrist/MCP) postures of the EDC session are illustrated on panels e and f, respectively.

Figure 2 - Description of the processing with the derivation of muscle behaviour (force-activation and length-force) relationships for each ramp (left column) and the derivation of individual ForceLength-Activation relationships at different activation levels to develop a Force-LengthActivation relationship (right column).

Figure 3 - Description of the process to compare the three EMG-driven musculoskeletal (MSK) models (a) and the general principle of muscle force and belly length estimation in the EMGdriven MSK models (b). IND: model based on individual Force-Length-Activation relationships; AVE: model based on average Force-Length-Activation relationships; NoFL: model based on average Force-Length-Activation relationships but only based on the maximal isometric force scaled by activation level. 
Figure 4 - Comparison of architectural properties obtained in this study for the ECR and EDC muscle groups with those directly measured on individual muscle bellies during dissection studies. EDCI, EDCM, EDCR and EDCL correspond to the index, middle, ring and little finger compartment of EDC, respectively. Data from Lieber(1990-92) [46, 47] correspond to mean \pm 1 standard deviation from 5 to 8 specimens whereas Mirakhorlo(2016) [20], Goislard(2018) and Kerkhof(2018): [21] reported data for a single specimen. The optimal fibre length in the current study was estimated by multiplying the optimal belly length $\left(L_{o p t}\right)$ by the index of architecture at maximal activation $\left(i_{a}^{\text {opt }}\right)$ [45]. The optimal fibre length values from Lieber(199092) were scaled to an optimal sarcomere length of $2.7 \mu \mathrm{m}[64]$ to be comparable with the other dissection studies. The maximal isometric force for all dissection studies was calculated by multiplying the sum of the PCSA of the muscles in the group by a maximal muscle stress value of $\left.35 \mathrm{~N} / \mathrm{cm}^{2} \llbracket 1,12\right\rceil$.

Figure $5-3 \mathrm{D}$ representation of the average Force-Length-Activation relationships of the population for the ECR (left panel) and EDC (right panel) muscles. Muscle force and belly length are normalized by the maximal isometric force at $F_{0}^{\text {max }}$, i.e. at $a^{m}=1$, and by the reference belly length $L_{r}^{m}$, i.e. at rest in neutral posture.

Figure 6 - Evolution of the parameters of the Force-Length-Activation relationship $\left(F_{0}, L_{0}, i_{a}\right)$ with the activation level $a^{m}$. Maximal isometric force and optimal belly length are normalized by the maximal isometric force at $a^{m}=1\left(F_{0}^{\max }\right)$ and by the reference belly length $L_{r}^{m}$, i.e. at rest in neutral posture. The lines represent the average curve of the whole population. The mean and standard deviations of the data from all individual relationships is represented by asterisk and cross signs, and shaded areas, respectively

Figure $7-$ Mean $\pm 1 \mathrm{SD}$ values of the normalized root mean square error of estimated muscle 
forces (RMSE; upper panels) and the muscle belly strain ( $\varepsilon^{m}$, middle panels) obtained with the three versions of the EMG-driven model as well as the maximum measured torque $\left(\tau_{j}^{\max }\right.$ with $\mathrm{j}=\{$ WR for wrist; MCP, for metacarpophalangeal joint $\}$ ) during the sawtooth contractions for the ECR (right panels) and EDC (left panels) muscles. IND: model based on individual Force-LengthActivation relationships; AVE: model based on average Force-Length-Activation relationships; NoFL: model based on average Force-Length-Activation relationships but only scaling the maximal isometric force by activation level, i.e. muscle always at optimal length

Figure 8 - Average Force-Length-Activation relationships for the sample population for both extensors (upper panels) along with the results of Hauraix et al. [33] for hand flexors (lower panels; FCR: flexor carpi radialis ; FDS: flexor digitorum superficialis). Muscle force and belly length are normalized by maximal isometric force and optimal belly length at maximal activation $\left(a^{m}=1\right)$. The colours correspond to different levels of activation, namely $1,0.75,0.5,0.25$ and 0 . The thin parts represent the theoretical force-length relationships for all belly length and the bold part symbolises the portions reached by each muscle for the finger and wrist range of motion tested in the experiment. For extrinsic muscles (EDC and FDS), the solid thick line indicates the portion reached when moving the fingers only with the wrist in neutral posture, and the dashed thick line indicates the supplementary shortening or lengthening when moving the wrist only at the extremities of the finger range of motion. The circle marker corresponds to the point where maximal isometric force is reached for different activation levels. The other markers indicate to which length the ramp (crosses) and sawtooth (diamond and star) postures were tested. The diamond marker corresponds to the neutral posture, i.e. $0^{\circ}$ of wrist and MCP flexion-extension. 


\section{Figures}
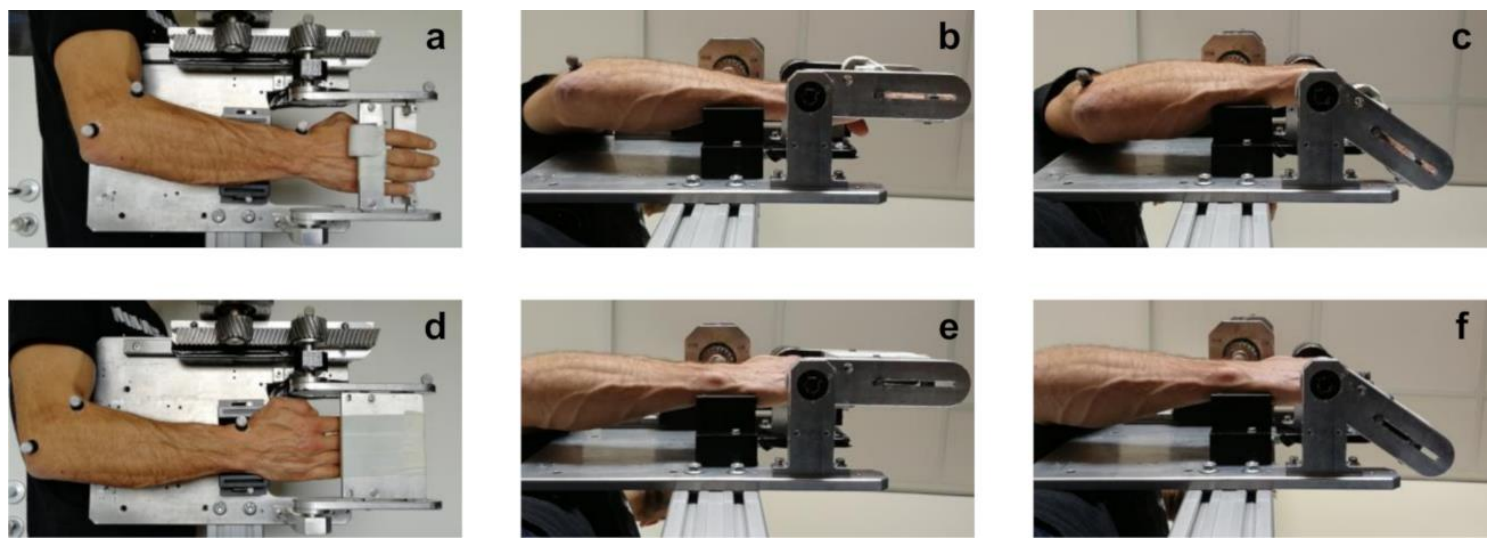

Figure 1 - Photographs illustrating the position of the hand in the ergometer and the different plates on which participants exerted extension moments for the ECR (panels a, b and c) and EDC sessions (d, e and f). For the ECR session (a), the participants aligned the wrist joint with the measurement axis and applied the extension moment using the dorsal aspect of the hand palm. The hand position for the 0 and the $50^{\circ}$ postures of the ECR session are illustrated on panels $b$ and c, respectively. For the EDC session (d), the participants aligned their MCP joints with the measurement axis and applied the extension moment using the dorsal aspect of the fingers. The hand position for the $0 / 0$ and the $0 / 50$ (Wrist/MCP) postures of the EDC session are illustrated on panels e and f, respectively. 


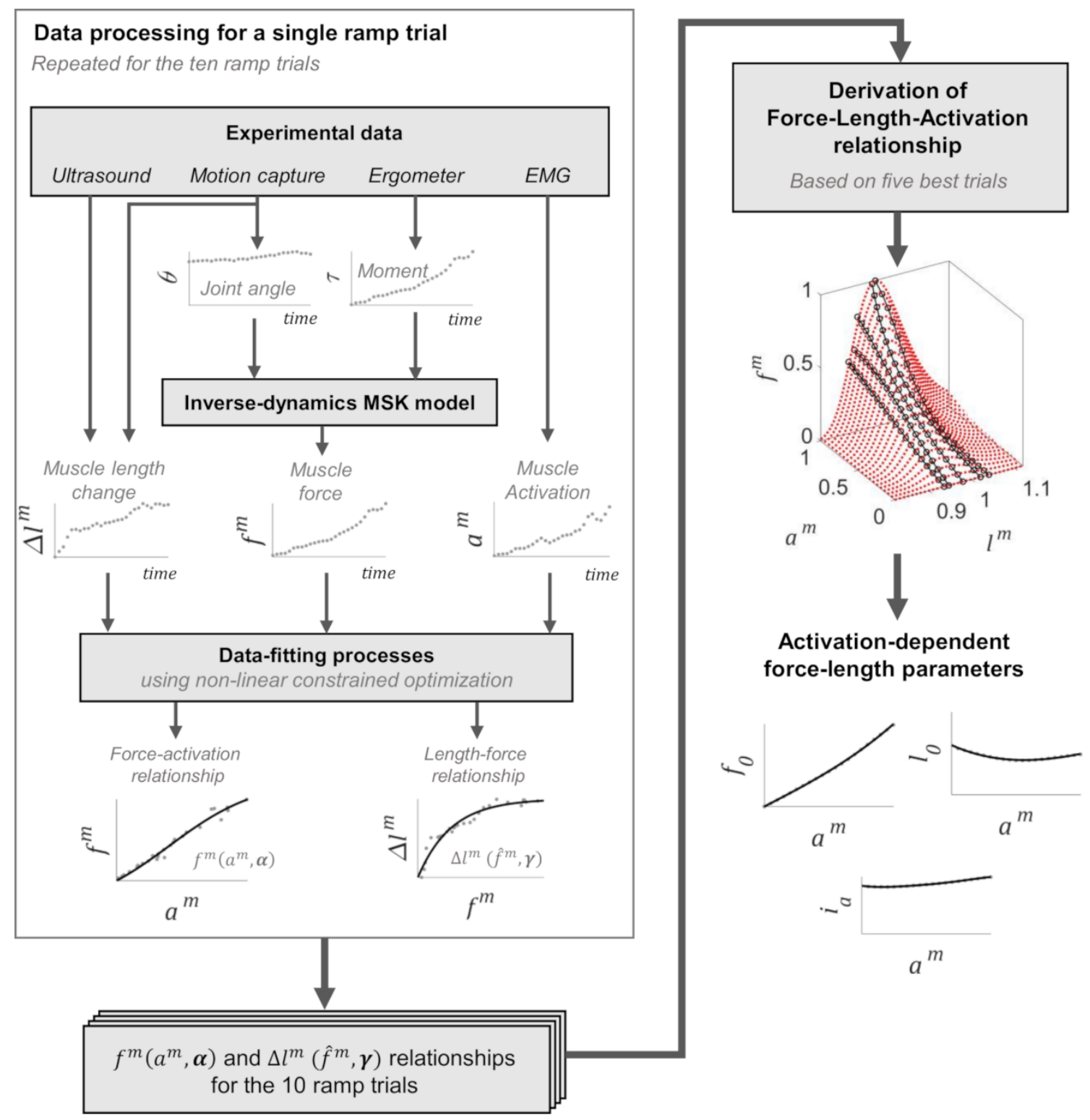

Figure 2 - Description of the processing with the derivation of muscle behaviour (force-activation and length-force) relationships for each ramp (left column) and the derivation of individual ForceLength-Activation relationships at different activation levels to develop a Force-LengthActivation relationship (right column). 


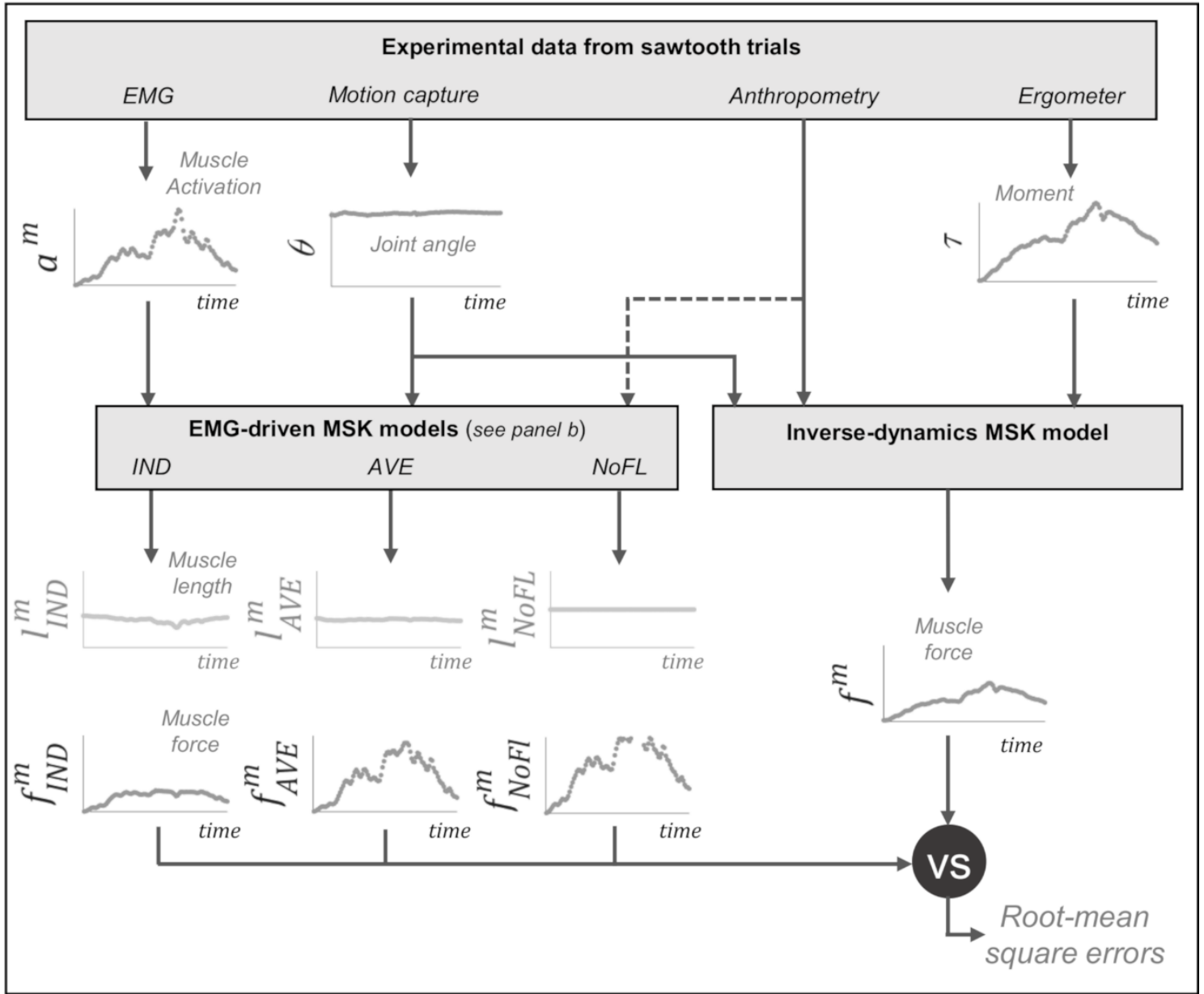

b Reference muscle- Joint Muscle tendon unit length angle Activation Principle of EMG-driven MSK model

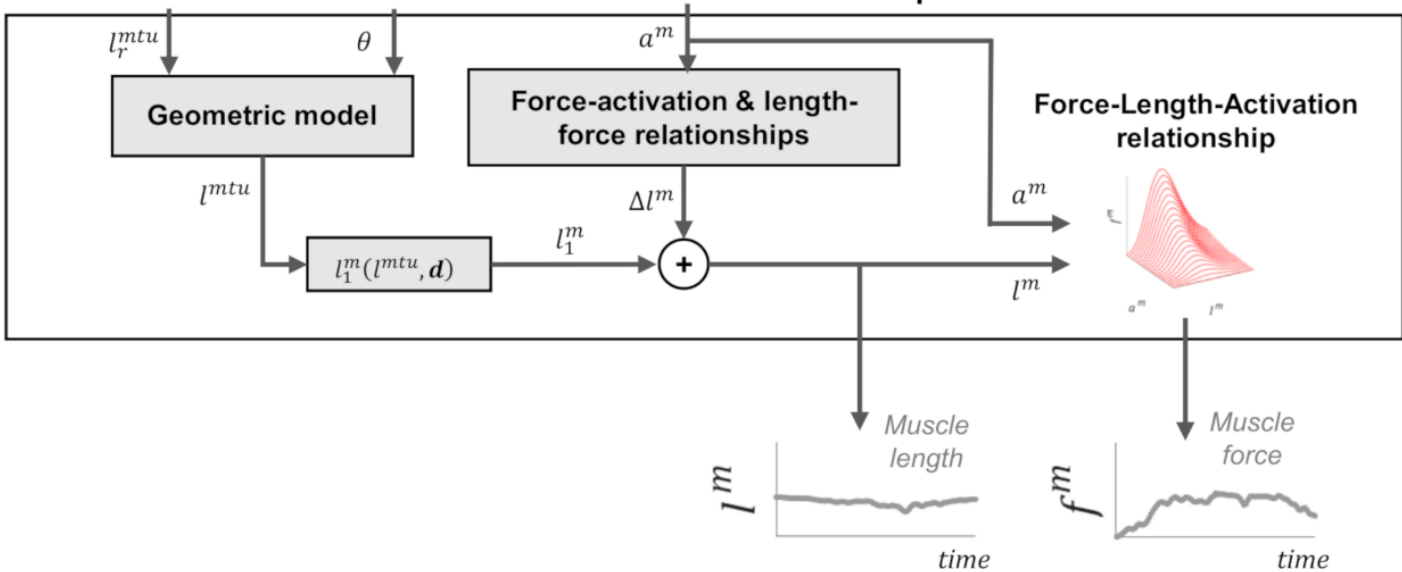

Figure 3 - Description of the process to compare the three EMG-driven musculoskeletal (MSK) models (a) and the general principle of muscle force and belly length estimation in the EMGdriven MSK models (b). IND: model based on individual Force-Length-Activation relationships; AVE: model based on average Force-Length-Activation relationships; NoFL: model based on 
average Force-Length-Activation relationships but only based on the maximal isometric force scaled by activation level.
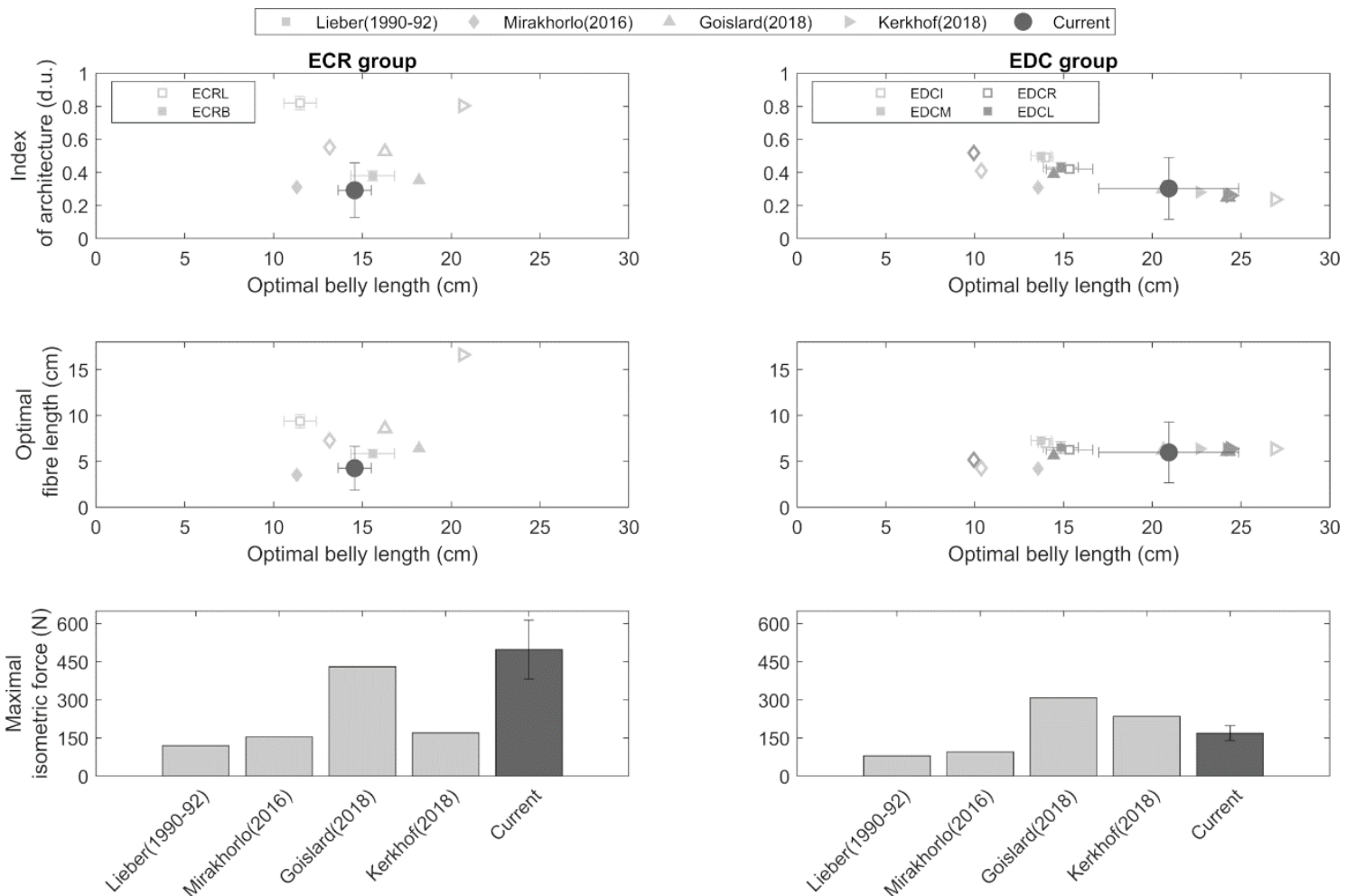

Figure 4 - Comparison of architectural properties obtained in this study for the ECR and EDC muscle groups with those directly measured on individual muscle bellies during dissection studies. EDCI, EDCM, EDCR and EDCL correspond to the index, middle, ring and little finger compartment of EDC, respectively. Data from Lieber(1990-92) [46, 47] correspond to mean \pm 1 standard deviation from 5 to 8 specimens whereas Mirakhorlo(2016) [20], Goislard(2018) [19] and Kerkhof(2018): [21] reported data for a single specimen. The optimal fibre length in the current study was estimated by multiplying the optimal belly length $\left(L_{o p t}\right)$ by the index of architecture at maximal activation $\left(i_{a}^{\text {opt }}\right)$ [45]. The optimal fibre length values from Lieber(199092) were scaled to an optimal sarcomere length of $2.7 \mu \mathrm{m}$ [64] to be comparable with the other dissection studies. The maximal isometric force for all dissection studies was calculated by multiplying the sum of the PCSA of the muscles in the group by a maximal muscle stress value of $35 \mathrm{~N} / \mathrm{cm}^{2}\lceil 1,12]$. 

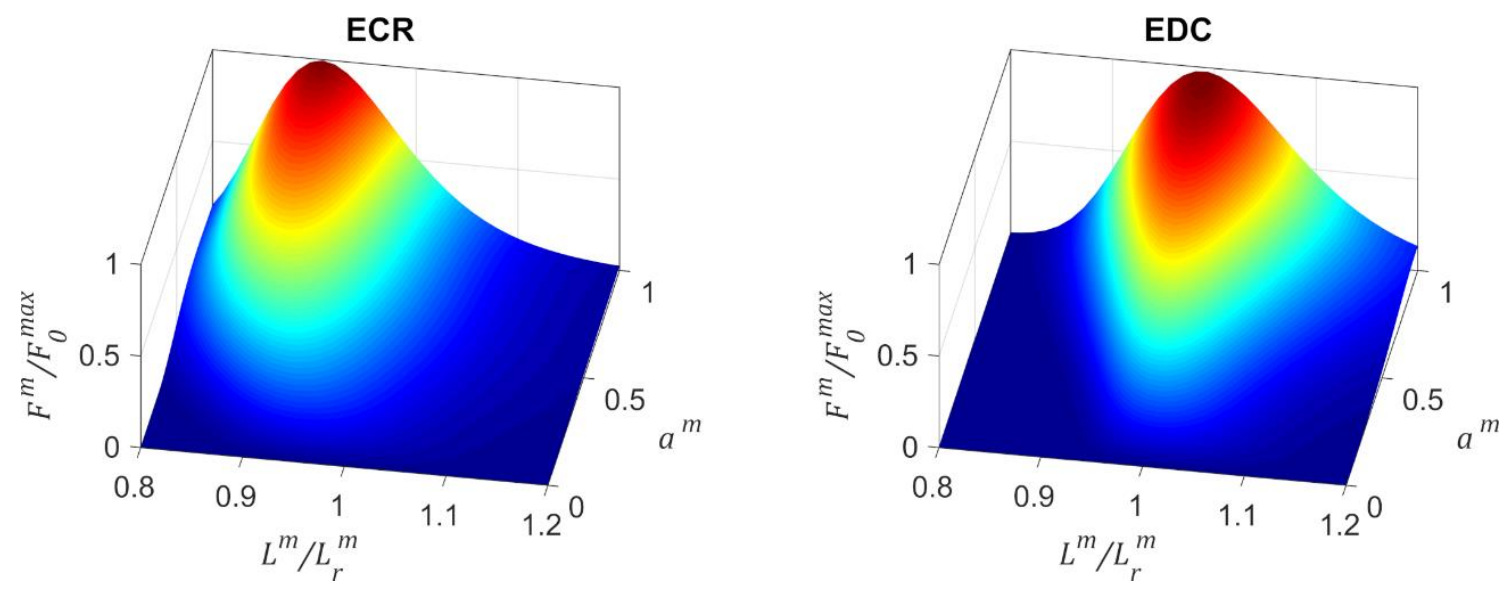

Figure $5-3 \mathrm{D}$ representation of the average Force-Length-Activation relationships of the population for the ECR (left panel) and EDC (right panel) muscles. Muscle force and belly length are normalized by the maximal isometric force at $F_{0}^{\max }$, i.e. at $a^{m}=1$, and by the reference belly length $L_{r}^{m}$, i.e. at rest in neutral posture. 

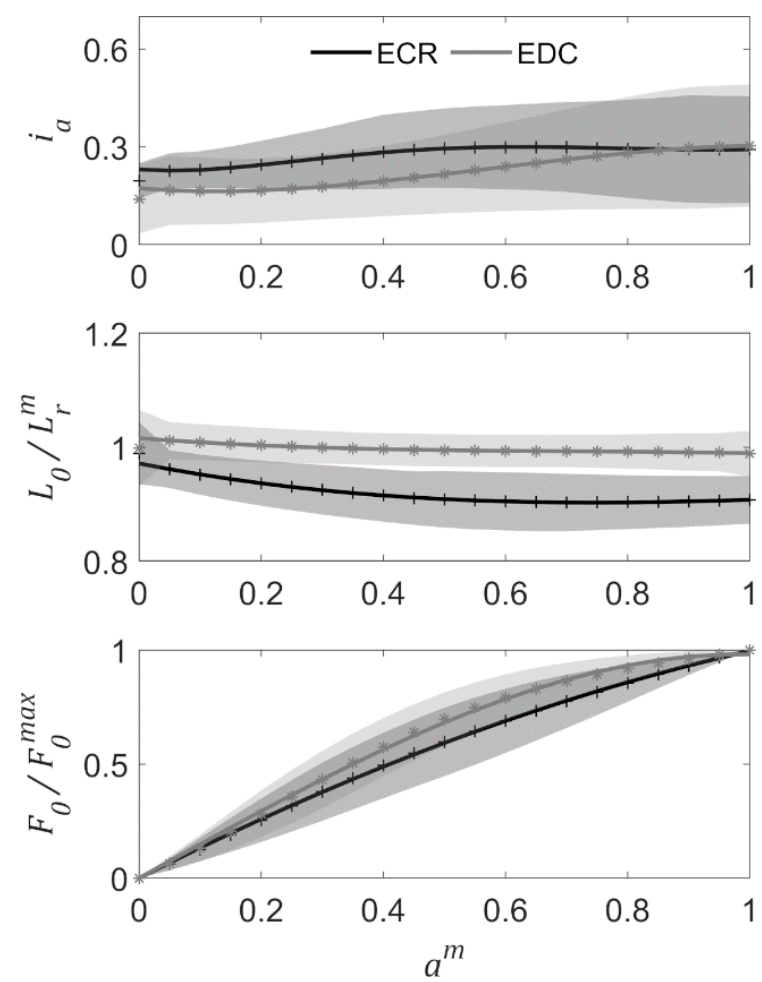

Figure 6 - Evolution of the parameters of the Force-Length-Activation relationship $\left(F_{0}, L_{0}, i_{a}\right)$ with the activation level $a^{m}$. Maximal isometric force and optimal belly length are normalized by the maximal isometric force at $a^{m}=1\left(F_{0}^{\max }\right)$ and by the reference belly length $L_{r}^{m}$, i.e. at rest in neutral posture. The lines represent the average curve of the sample population. The mean and standard deviations of the data from all individual relationships is represented by asterisk and cross signs, and shaded areas, respectively. 
ECR
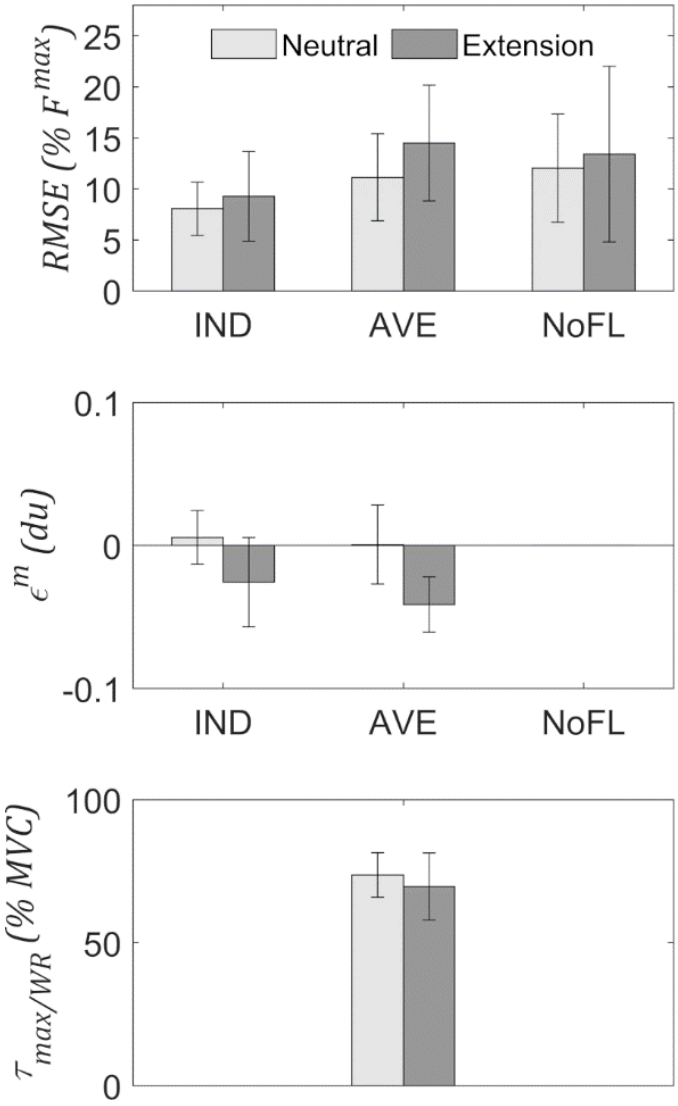

EDC
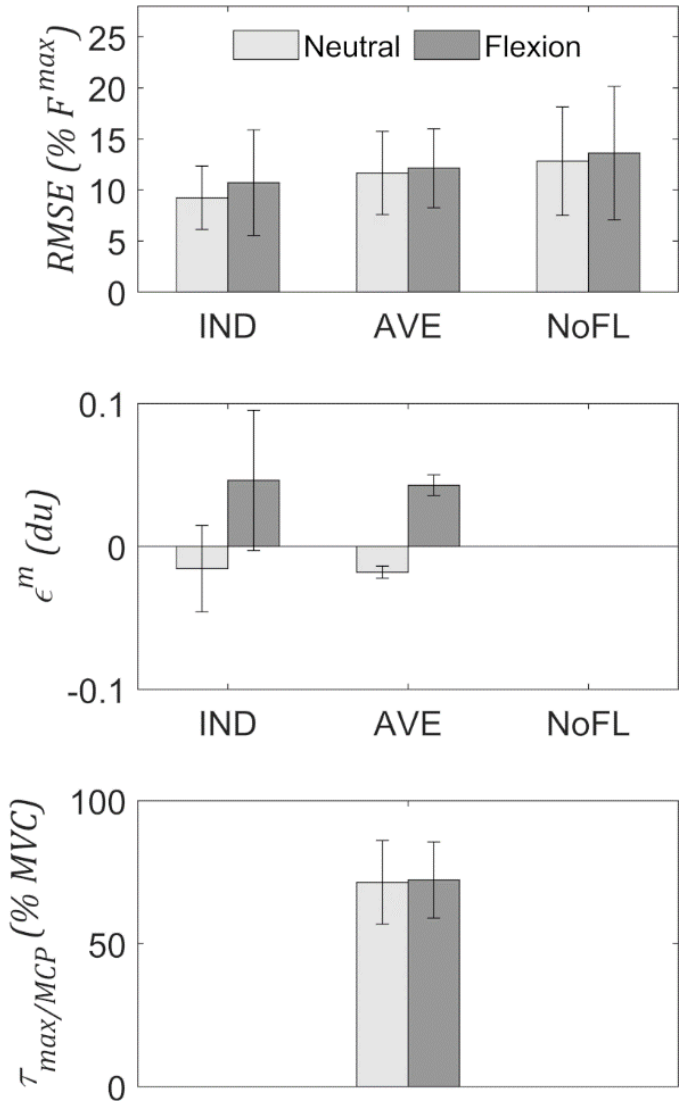

Figure $7-$ Mean \pm 1 SD values of the normalized root mean square error of estimated muscle forces (RMSE; upper panels) and the muscle belly strain ( $\varepsilon^{m}$, middle panels) obtained with the three versions of the EMG-driven model as well as the maximum measured torque ( $\tau_{j}^{\max }$ with $j=\{$ WR for wrist; MCP, for metacarpophalangeal joint $\}$ ) during the sawtooth contractions for the ECR (right panels) and EDC (left panels) muscles. IND: model based on individual Force-LengthActivation relationships; AVE: model based on average Force-Length-Activation relationships; NoFL: model based on average Force-Length-Activation relationships but only scaling the maximal isometric force by activation level, i.e. muscle always at optimal length 

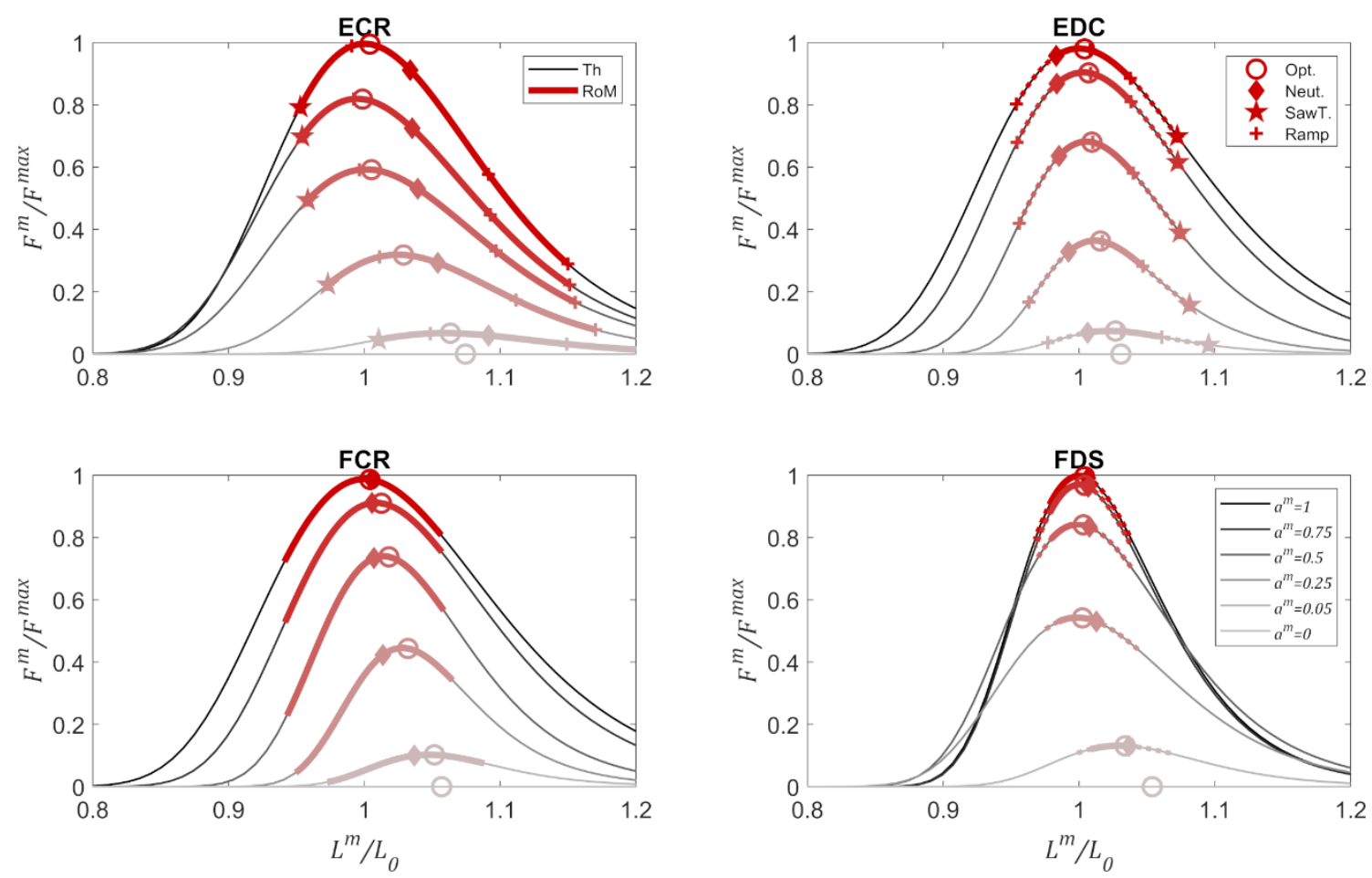

Figure 8 - Average Force-Length-Activation relationships for the sample population for both extensors (upper panels) along with the results of Hauraix et al. [33] for hand flexors (lower panels; FCR: flexor carpi radialis ; FDS: flexor digitorum superficialis). Muscle force and belly length are normalized by maximal isometric force and optimal belly length at maximal activation $\left(a^{m}=1\right)$. The colours correspond to different levels of activation, namely $1,0.75,0.5,0.25$ and 0 . The thin parts represent the theoretical force-length relationships for all belly length and the bold part symbolises the portions reached by each muscle for the finger and wrist range of motion tested in the experiment. For extrinsic muscles (EDC and FDS), the solid thick line indicates the portion reached when moving the fingers only with the wrist in neutral posture, and the dashed thick line indicates the supplementary shortening or lengthening when moving the wrist only at the extremities of the finger range of motion. The circle marker corresponds to the point where maximal isometric force is reached for different activation levels. The other markers indicate to which length the ramp (crosses) and sawtooth (diamond and star) postures were tested. The diamond marker corresponds to the neutral posture, i.e. $0^{\circ}$ of wrist and MCP flexion-extension. 


\section{Tables}

Table 1 - Measured wrist and MCP torque as well as estimated variables describing the forcegenerating capacities and the architecture of both muscles.

\begin{tabular}{lccc} 
& Wrist & MCP & $\boldsymbol{P}$ \\
\hline$\tau^{\max }(\mathrm{Nm})$ & $14.1 \pm 3.1$ & $3.5 \pm 0.9$ & $<0.001$ \\
\hline & ECR & EDC & \\
\hline$F_{0}^{\max }(\mathrm{N})$ & $498 \pm 115$ & $169 \pm 29$ & $<0.001$ \\
$L_{r}^{m t u}(\mathrm{~cm})$ & $31.4 \pm 1.5$ & $37.1 \pm 1.6$ & $<0.001$ \\
$L_{r}^{m}(\mathrm{~cm})$ & $16.1 \pm 0.01$ & $21.1 \pm 3.7$ & 0.0019 \\
$L_{\text {opt }}(\mathrm{cm})$ & $14.6 \pm 0.01$ & $20.9 \pm 3.9$ & $<0.001$ \\
$l_{\text {opt }}\left(\% L_{r}^{m}\right)$ & $90.8 \pm 4.0$ & $98.9 \pm 3.8$ & $<0.001$ \\
$i_{a}^{\text {opt }},($ d.u. $)$ & $0.29 \pm 0.17$ & $0.30 \pm 0.19$ & 0.89 \\
\hline MCP: metacarpophalangeal & joint; ECR: & Extensor \\
Carpi $\quad$ Radialis; & EDC: & Extensor & Digitorum
\end{tabular}

Communis. Mathematical symbols described in the text. 


\section{Electronic Supplementary materials}

Electronic Supplementary material 1 - pdf document providing the data associated to the sample population Force-Length-Activation relationships for both muscles. 